\title{
CONSTRUCTION OF THE VALUE FUNCTION AND OPTIMAL RULES IN OPTIMAL STOPPING OF ONE-DIMENSIONAL DIFFUSIONS
}

\author{
KURT HELMES, ${ }^{*}$ Humboldt-Universität zu Berlin \\ RICHARD H. STOCKBRIDGE,** University of Wisconsin-Milwaukee
}

\begin{abstract}
A new approach to the solution of optimal stopping problems for one-dimensional diffusions is developed. It arises by imbedding the stochastic problem in a linear programming problem over a space of measures. Optimizing over a smaller class of stopping rules provides a lower bound on the value of the original problem. Then the weak duality of a restricted form of the dual linear program provides an upper bound on the value. An explicit formula for the reward earned using a two-point hitting time stopping rule allows us to prove strong duality between these problems and, therefore, allows us to either optimize over these simpler stopping rules or to solve the restricted dual program. Each optimization problem is parameterized by the initial value of the diffusion and, thus, we are able to construct the value function by solving the family of optimization problems. This methodology requires little regularity of the terminal reward function. When the reward function is smooth, the optimal stopping locations are shown to satisfy the smooth pasting principle. The procedure is illustrated using two examples.
\end{abstract}

Keywords: Optimal stopping; linear programming; duality; nonlinear optimization; value function; one-dimensional diffusion

2000 Mathematics Subject Classification: Primary 60G40

Secondary $60 \mathrm{~J} 60$

\section{Introduction}

In this paper we develop a new approach to the solution of optimal stopping problems for one-dimensional diffusions. By imbedding the optimal stopping problem in an infinitedimensional linear program (LP) and examining its dual, a nonlinear optimization problem and a semi-infinite LP are derived from which the value function is able to be constructed for a wide class of reward functions.

We consider a one-dimensional diffusion $X$ which satisfies the stochastic differential equation

$$
\mathrm{d} X(t)=\mu(X(t)) \mathrm{d} t+\sigma(X(t)) \mathrm{d} W(t), \quad X(0)=x,
$$

\footnotetext{
Received 16 December 2008; revision received 15 December 2009.

* Postal address: Institut für Operations Research, Humboldt-Universität zu Berlin, Germany.

Email address: helmes@wiwi.hu-berlin.de

** Postal address: Department of Mathematical Sciences, University of Wisconsin-Milwaukee, Milwaukee, WI 53201-0413, USA. Email address: stockbri@uwm.edu

This research has been supported in part by the US National Security Agency under Grant Agreement Number H9823009-1-0002. The United States Government is authorized to reproduce and distribute reprints notwithstanding any copyright notation herein.
} 
in some interval $\left(x_{l}, x_{r}\right)$. The objective is to select a time $\tau$ so as to maximize

$$
J(\tau ; x):=\mathrm{E}\left[\int_{0}^{\tau} \mathrm{e}^{-\alpha s} r(X(s)) \mathrm{d} s+\mathrm{e}^{-\alpha \tau} g(X(\tau)) \mathbf{1}_{\{\tau<\infty\}}\right] .
$$

In this expression, $\alpha>0$ denotes a discount rate, $r$ is a running reward function, and $g$ represents the reward obtained at the terminal time. The need for a discount factor arises when the time frame for stopping is such that alternative investment possibilities affect the value of the reward earned. This, for example, will be the case for many perpetual options and for many applied problems such as the harvesting of renewable resources. For example, in forest harvesting, $r$ might represent the amenity value or carbon credit of the forest while $g$ would give the value derived from harvesting.

Typically, we are interested in determining both an optimal stopping time $\tau^{*}$ and the value function

$$
V(x)=\sup _{\tau} J(\tau ; x)
$$

It is helpful to observe that $V$ is a function of the initial position of the diffusion $X$. This will become important when the problem is imbedded in a family of LPs parameterized by $x$. We present a careful formulation of the optimal stopping problem in Section 1.1 and impose further technical conditions in Sections 2, 4, and 5 that guarantee finiteness of the discounted reward and existence of optimal stopping rules.

Optimal stopping of stochastic processes has a long history which has resulted in several solution approaches. Two excellent surveys of the general theory of optimal stopping are El Karoui [6] and Zabczyk [16]. The book by Shiryaev [15] approaches a nondiscounted version of the above problem in which $r \equiv 0$ by seeking the minimal excessive majorant of $g$. This minimal excessive function is the value function $V$ and an optimal stopping rule is determined by stopping when the process $X$ first hits a point $a$ where $V(a)=g(a)$. The key to this solution technique is identifying the minimal excessive function $V$ along with the set $\{a: V(a)=g(a)\}$. The recent book by Peskir and Shiryaev [12] relates the solution of optimal stopping problems to the solution of free boundary problems and uses the terminology of superharmonic functions in place of excessive functions. The authors considered more general problems that involve processes with jumps and include rewards based on the supremum of the process $X$ as well as running and terminal rewards. For continuous processes, they employed the method of smooth pasting; that is, they sought to determine a (not necessarily connected) open continuation region $\mathcal{C}$, a (not necessarily connected) closed stopping region $\delta$, and a function $V$ for which

(i) $\mathcal{C} \cap s=\varnothing, \mathcal{C} \cup s=\left[x_{l}, x_{r}\right]$,

(ii) $V \in C^{1}\left[x_{l}, x_{r}\right]$, with $\left.V\right|_{\mathcal{C}} \in C^{2}(\mathcal{C})$,

(iii) $A V(y)-\alpha V(y)+r(y)=0$ for all $y \in \mathcal{C}$, and

(iv) $V(y)=g(y)$ for all $y \in \delta$,

where $A$ in (iii) denotes the generator of $X$ so

$$
A V(y)=\mu(y) V^{\prime}(y)+\frac{1}{2} \sigma^{2}(y) V^{\prime \prime}(y) .
$$

The moniker 'smooth pasting' arises from the fact that we seek to paste the solution of the differential equation in the region $\mathcal{C}$ from (iii) to the function $g$ on the set $\delta$ with the function so defined being continuously differentiable at the boundary points $\& \cap \overline{\mathcal{C}}$. When the process has 
jumps, the condition of smooth pasting is relaxed to continuous pasting, though smooth fit may also hold [1]. Optimal stopping of diffusion processes using smooth pasting is also discussed by Salminen [14], Dayanik and Karatzas [4], and in the text of Øksendal [11, Chapter 10].

The book by Dynkin [5, pp. 146, 155] and the recent paper by Dayanik and Karatzas [4] show that the excessive functions are characterized as concave functions in a generalized sense. The problem of determining the minimum excessive function which majorizes the reward function is therefore recast as a problem of finding the minimum generalized concave function which majorizes the reward function. The paper [4] illustrates this approach on a number of optimal stopping problems.

As indicated in the first paragraph, this paper approaches the optimal stopping problem quite differently. As will be seen in Section 1.1, the problem formulation optimizes over all stopping times. Sections 2 to 4, however, examine carefully the optimal stopping problem over a restricted class of stopping times. The stochastic problem is imbedded in an infinitedimensional LP (Section 2). We then optimize over an even smaller class of stopping times in Section 3, specifically the two-point hitting times, and relax the constraints to form an auxiliary linear problem. A dual LP is derived for which a weak duality relationship exists between the LPs. In Section 4, strong duality between the problems and sufficiency of a two-point hitting rule are proven. The result of strong duality is that the restricted stopping problem is reformulated as an explicit nonlinear optimization problem and as a semi-infinite LP, both of which can be used to determine the value. When the (modified) terminal reward function is smooth, the smooth pasting principle follows from the necessary conditions for optimality in the nonlinear problem. The unrestricted optimal stopping problem is then analyzed in Section 5, in which it is shown that the values of the unrestricted and restricted problems are the same, and, as a result, it follows that optimizing over two-point stopping rules determines optimal stopping rules. This solution technique is then illustrated in Section 6 using two examples.

The paper by Alvarez [2] is closest to our approach in that he analyzed a nonlinear program and demonstrated that the principle of smooth fit is a consequence of his optimization procedure. His solution approach is global, whereas the approach in this paper is local and allows the value function to be constructed. In addition, our paper provides an explicit reformulation for the running reward term and, hence, we identify a simple nonlinear optimization problem. The key to our result is Theorem 4.2, which proves strong duality between all our LPs.

\subsection{Detailed formulation}

Let the coefficients $\mu$ and $\sigma$ in (1.1) be continuous. Furthermore, assume that $\mu$ and $\sigma$ are such that $X$ is a weak solution of (1.1) while $X(t) \in\left(x_{l}, x_{r}\right)$ (see [7, Section 5.3, p. 291] or [13, V.16.1, p. 149] for details) and that the solution to (1.1) is unique in distribution. This uniqueness implies that the martingale problem for $A$ is well posed and, hence, that $X$ is a strong Markov process (see [7, Theorem 4.4.2, p. 184] or [13, V.21.2, p. 162]). We denote the filtration for the weak solution by $\left\{\mathcal{F}_{t}\right\}$.

Each of the boundary points $x_{l}$ and $x_{r}$ can be classified as a natural, an entrance, or an exit boundary point depending on the characteristics of the drift coefficient $\mu(\cdot)$ and diffusion coefficient $\sigma(\cdot)$ (see [3, II.10, pp. 14-19] or [10, pp. 128-131]). When a point is both an exit and an entrance boundary point, the point is called nonsingular and the diffusion is not determined uniquely. We therefore assume that the boundary points are singular. When $x_{l}$ is a natural boundary point and $x>x_{l}$, the process $X$ will not hit $x_{l}$ in finite time (a.s.). The point $x_{l}$ is thus not part of the state space for $X$. When $x_{l}$ is an entrance-not-exit boundary point and $x \geq x_{l}$, $X(t) \in\left(x_{l}, x_{r}\right]$ (a.s.) for all $t>0$, so when $x=x_{l}$, the process immediately enters the interval 
$\left(x_{l}, x_{r}\right)$ and never exits at $x_{l}$. When $x_{l}$ is an exit-not-entrance boundary point, there is a positive probability that $X$ hits $x_{l}$ in finite time. Similar statements apply to $x_{r}$. When $x_{l}=-\infty$ and/or $x_{r}=\infty$, we require these points to be natural boundaries of the diffusion, which implies that the diffusion is nonexplosive.

In light of the hitting behavior of the boundary points, for simplicity, we assume that the initial position $x$ satisfies $x \in\left(x_{l}, x_{r}\right)$. We emphasize that throughout this paper, $x$ is reserved to be the initial value of the diffusion. It will never be used as a dummy variable in any expression.

As already noted, in the case where either $x_{l}$ or $x_{r}$ is an exit boundary point, $X$ will have positive probability of hitting the boundary in finite time, so the choices of stopping times cannot exceed this hitting time. Let $\zeta=\inf \left\{t>0: X(t) \notin\left(x_{l}, x_{r}\right)\right\}$ denote the time at which the process exits $\left(x_{l}, x_{r}\right)$. The running reward function $r$, defined on $\left(x_{l}, x_{r}\right)$, is required to satisfy

$$
\mathrm{E}\left[\int_{0}^{\zeta} \mathrm{e}^{-\alpha s}|r(X(s))| \mathrm{d} s\right]<\infty
$$

conditions on the terminal reward function $g$ are specified indirectly in Conditions 2.1, 4.1, and 5.1. The objective is to select an $\left\{\mathcal{F}_{t}\right\}$-stopping time $\tau \leq \zeta$ so as to maximize (1.2). Note that, when $X(t) \in\left(x_{l}, x_{r}\right)$ for all $t$, by convention, $\zeta=\infty$ and no restriction is placed on the stopping times. Let $\mathcal{A}$ denote the set of admissible $\left\{\mathcal{F}_{t}\right\}$-stopping times; this means that $\mathcal{A}$ has the additional constraint that $\tau \leq \zeta$ when either boundary point is an exit-not-entrance boundary point, but otherwise is unrestricted. To be specific, $V$ defined in (1.3) is taken to be the supremum over $\tau \in \mathcal{A}$.

We note that $\left\{\mathcal{F}_{t}\right\}$ is the filtration associated with the weak solution to (1.1) so it may contain more information than that arising from the observations of the process $X$. Since the stopping times $\tau \in \mathcal{A}$ are $\left\{\mathscr{F}_{t}\right\}$-measurable, these may in principle be determined using information contained in $\left\{\mathcal{F}_{t}\right\}$ that is not generated by $X$. Our results nevertheless show that optimal stopping rules exist within the subclass of hitting times of the process.

Our definition of $\left\{\mathcal{F}_{t}\right\}$-stopping times follows that of Ethier and Kurtz [7, p. 51] and allows stopping times to take value $\infty$. Peskir and Shiryaev [12] referred to these random variables as Markov times and reserved the term stopping time to be those Markov times which are finite almost surely. We allow the stopping times to be infinite on a set of positive probability, in which case the decision is not to stop and receive any terminal reward. Clearly, this decision should not be, and is not, rewarded when there is no running reward and the terminal reward is positive.

As a preliminary step, we wish to eliminate the running reward from the objective by adjusting the terminal reward function. To do so, we require the following condition.

Condition 1.1. The reward function $r$ and the diffusion are such that there exists a function $f_{r}$ with the property that, for all $\tau \in \mathcal{A}$ and initial values $x \in\left(x_{l}, x_{r}\right)$,

$$
\mathrm{E}\left[\int_{0}^{\tau} \mathrm{e}^{-\alpha s} r(X(s)) \mathrm{d} s\right]=\mathrm{E}\left[\mathrm{e}^{-\alpha \tau} f_{r}(X(\tau)) \mathbf{1}_{\{\tau<\infty\}}\right]-f_{r}(x) .
$$

The next proposition indicates that this condition is satisfied for a large class of reward functions. In this proposition, we use the notation $\mathrm{E}_{x}[\cdot]$ to emphasize the initial value of $X$, which will be of importance for the application of the strong Markov property. Some additional preparation is necessary for the proof of the proposition. 
Note that the generator of the discounted process $\left\{\mathrm{e}^{-\alpha t} X(t): t \geq 0\right\}$ is $A f(y)-\alpha f(y)=$ $\frac{1}{2} \sigma^{2}(y) f^{\prime \prime}(y)+\mu(y) f^{\prime}(y)-\alpha f(y)$. It is well known (see [3, II.10, pp. 18, 19] or [10, pp. 128-131]) that, under the conditions assumed in this paper, the eigenfunction equation

$$
A f=\alpha f
$$

has a positive, strictly decreasing solution $\phi$ and a nonnegative, strictly increasing solution $\psi$ as its fundamental solutions. (Both $\phi$ and $\psi$ depend on $\alpha$; since we assume $\alpha$ is fixed, we do not use notation that indicates this dependence.) The functions $\phi$ and $\psi$ are unique up to a multiplicative factor. Furthermore, $\phi\left(x_{l}+\right)=\infty$ and $\psi\left(x_{l}+\right) \geq 0$ when $x_{l}$ is either a natural or an entrance-not-exit boundary of $X$ and $\phi\left(x_{l}+\right) \in(0, \infty)$ when $x_{l}$ is an exit-not-entrance boundary point. Similar comments apply to $x_{r}$ with the roles of $\phi$ and $\psi$ reversed.

Proposition 1.1. Let $r$ be bounded and continuous on $\left(x_{l}, x_{r}\right)$. Then Condition 1.1 holds.

Proof. Let $\tau \in \mathcal{A}$ and $x \in\left(x_{l}, x_{r}\right)$ be chosen arbitrarily. Set $r\left(x_{l}\right)=0=r\left(x_{r}\right)$ so that no reward is accrued once the process $X$ is absorbed at the boundary. Then

$$
\mathrm{E}_{x}\left[\int_{0}^{\tau} \mathrm{e}^{-\alpha s} r(X(s)) \mathrm{d} s\right]=\mathrm{E}_{x}\left[\int_{0}^{\infty} \mathrm{e}^{-\alpha s} r(X(s)) \mathrm{d} s-\mathbf{1}_{\{\tau<\infty\}} \int_{\tau}^{\infty} \mathrm{e}^{-\alpha s} r(X(s)) \mathrm{d} s\right] .
$$

Define $f_{r}(x)=-\mathrm{E}_{x}\left[\int_{0}^{\infty} \mathrm{e}^{-\alpha s} r(X(s)) \mathrm{d} s\right]$. Then $f_{r}$ is well defined and has an integral representation in terms of the function $r$, and the Green kernel and the speed measure of the process (see [3, II.1.4]). This representation shows that $f_{r}$ is twice differentiable and is a particular solution of $A f-\alpha f=r$. Furthermore, by the strong Markov property we get

$$
\begin{aligned}
\mathrm{E}_{x}\left[\mathbf{1}_{\{\tau<\infty\}} \int_{\tau}^{\infty} \mathrm{e}^{-\alpha s} r(X(s)) \mathrm{d} s\right] \\
=\mathrm{E}_{x}\left[\mathbf{1}_{\{\tau<\infty\}} \mathrm{E}_{x}\left[\int_{\tau}^{\infty} \mathrm{e}^{-\alpha s} r(X(s)) \mathrm{d} s \mid \mathcal{F}_{\tau}\right]\right] \\
=\mathrm{E}_{x}\left[\mathbf{1}_{\{\tau<\infty\}} \mathrm{e}^{-\alpha \tau} \mathrm{E}_{x}\left[\int_{0}^{\infty} \mathrm{e}^{-\alpha u} r(X(\tau+u)) \mathrm{d} u \mid X(\tau)\right]\right] \\
=\mathrm{E}_{x}\left[\mathbf{1}_{\{\tau<\infty\}} \mathrm{e}^{-\alpha \tau} \mathrm{E}_{X(\tau)}\left[\int_{0}^{\infty} \mathrm{e}^{-\alpha u} r(X(u)) \mathrm{d} u\right]\right] \\
=-\mathrm{E}_{x}\left[\mathbf{1}_{\{\tau<\infty\}} \mathrm{e}^{-\alpha \tau} f_{r}(X(\tau))\right],
\end{aligned}
$$

from which the assertion follows.

Under Condition 1.1, the reward obtained under an admissible stopping time $\tau \in \mathcal{A}$ is

$\mathrm{E}\left[\int_{0}^{\tau} \mathrm{e}^{-\alpha s} r(X(s)) \mathrm{d} s+\mathrm{e}^{-\alpha \tau} g(X(\tau)) \mathbf{1}_{\{\tau<\infty\}}\right]=\mathrm{E}\left[\mathrm{e}^{-\alpha \tau}\left(f_{r}+g\right)(X(\tau)) \mathbf{1}_{\{\tau<\infty\}}\right]-f_{r}(x)$.

Thus, using the function $f_{r}$ allows the replacement of the running reward of the objective function by suitably adjusting the terminal reward earned at time $\tau$ and shifting by the constant $-f_{r}(x)$. Since the constant shift is the same for each stopping rule $\tau \in \mathcal{A}$, it may be ignored for optimization purposes. Define $g_{r}=f_{r}+g$. The stopping problem is now one of maximizing

$$
J_{r}(\tau ; x):=\mathrm{E}\left[\mathrm{e}^{-\alpha \tau} g_{r}(X(\tau)) \mathbf{1}_{\{\tau<\infty\}}\right]
$$


over stopping times $\tau \in \mathcal{A}$. Denote the value function for this optimal stopping problem by

$$
V_{r}(x)=\sup _{\tau \in \mathcal{A}} J_{r}(\tau ; x)=\sup _{\tau \in \mathcal{A}} J(\tau ; x)+f_{r}(x) .
$$

In the sequel, we assume that, for each $\tau \in \mathcal{A}$, the random variable $\mathrm{e}^{-\alpha \tau} g_{r}(X(\tau)) \mathbf{1}_{\{\tau<\infty\}}$ is integrable.

The diffusion processes under consideration in this paper exclude reflection at a boundary and killing. The inclusion of reflected processes would require either restricting the domain of the test functions to capture the reflection or adding a second operator $B$ that adjusts the evolution of the process $X$ when reflection occurs. The latter approach adapts well to the linear programming methodology and is an example of one type of singular behavior [8]. The exclusion of killing is merely for convenience so as to clearly observe the effect discounting has on the problem. The generator $A$ for a killed diffusion is $A f(y)=\frac{1}{2} \sigma^{2}(y) f^{\prime \prime}(y)+\mu(y) f^{\prime}(y)-c(y) f(y)$, where $c \geq 0$ gives the state-dependent killing rate; when discounting is also included, the operator of interest is $A f(y)-\alpha f(y)=\frac{1}{2} \sigma^{2}(y) f^{\prime \prime}(y)+\mu(y) f^{\prime}(y)-(c(y)+\alpha) f(y)$. The key requirements for this paper are that (1.4) has decreasing and increasing solutions $\phi$ and $\psi$, respectively (see $[3, \mathbf{1 0}$, p. 18]), and that Condition 1.1 has a solution. Thus, the results of this paper easily extend to diffusions having state-dependent killing rates.

\section{LP problem and stopping rule analysis}

For models in which both boundaries $x_{l}$ and $x_{r}$ are exit-not-entrance points, the arguments of Sections 2, 3, and 4 hold without restrictions on the set of stopping times. For models in which one of $x_{l}$ or $x_{r}$ is either a natural or an entrance-not-exit boundary point, it is necessary to first restrict the optimization problem to a subclass $\mathcal{A}_{1}$ of stopping times. We then show in Section 5 that the optimal solution for the restricted problem is optimal for the unrestricted problem.

Suppose that $x_{l}$ is either a natural or entrance-not-exit boundary. Each stopping time $\tau$ in $\mathcal{A}_{1}$ satisfies the condition that there exists some $a_{\tau}$ with $x_{l}<a_{\tau} \leq x$ such that $X(t) \geq a_{\tau}$ for all $0 \leq t \leq \tau$. Similarly, when $x_{r}$ is either a natural or entrance-not-exit boundary point, for each stopping time $\tau \in \mathcal{A}_{1}$, there exists some $x \leq b_{\tau}<x_{r}$ for which $X(t) \leq b_{\tau}$ for $0 \leq t \leq \tau$. For the case that both $x_{l}$ and $x_{r}$ are natural or entrance-not-exit, both of these conditions must be satisfied and, hence, for $0 \leq t \leq \tau, a_{\tau} \leq X(t) \leq b_{\tau}$ for some $a_{\tau}$ and $b_{\tau}$. The choice of $a_{\tau}$ and $b_{\tau}$ depend on the particular stopping time $\tau \in \mathcal{A}_{1}$ and, hence, may differ for different $\tau$, but the requirement that $a_{\tau} \leq X(t) \leq b_{\tau}$ must hold for all $0 \leq t \leq \tau$.

\subsection{Derivation of the LP}

We take the initial position $x \in\left(x_{l}, x_{r}\right)$ to be arbitrary but fixed in the following discussion. We focus our arguments on natural or entrance-not-exit boundary points and consider functions on the open interval $\left(x_{l}, x_{r}\right)$; the case for exit boundary points involves using nice functions on the closed interval and considering times up to the lifetime $\zeta$ of the process.

Let $f \in C^{2}\left(x_{l}, x_{r}\right)$ have compact support. Since $X$ satisfies (1.1), an application of Itô's formula yields

$$
\mathrm{e}^{-\alpha t} f(X(t))=f(x)+\int_{0}^{t} \mathrm{e}^{-\alpha s}[A f(X(s))-\alpha f(X(s))] \mathrm{d} s+\int_{0}^{t} \mathrm{e}^{-\alpha s} f^{\prime}(X(s)) \mathrm{d} W(s) .
$$

Now let $\tau$ be any stopping time in $\mathcal{A}_{1}$. The optional sampling theorem [7, Theorem 2.2.13] 
implies that

$$
\begin{aligned}
\mathrm{e}^{-\alpha(t \wedge \tau)} f(X(t \wedge \tau))-f(x)-\int_{0}^{t \wedge \tau} \mathrm{e}^{-\alpha s}[A f(X(s))-\alpha f(X(s))] \mathrm{d} s \\
=\int_{0}^{t \wedge \tau} \mathrm{e}^{-\alpha s} f^{\prime}(X(s)) \mathrm{d} W(s),
\end{aligned}
$$

and so the left-hand side is a martingale. Since $\mu$ and $\sigma$ are bounded on compact sets, and $f$ and its derivatives are bounded, taking expectations in (2.1) and letting $t \rightarrow \infty$ yields Dynkin's formula (see, e.g. [10, p. 98])

$$
\mathrm{E}\left[\mathrm{e}^{-\alpha \tau} f(X(\tau)) \mathbf{1}_{\{\tau<\infty\}}-\int_{0}^{\tau} \mathrm{e}^{-\alpha s}[A f(X(s))-\alpha f(X(s))] \mathrm{d} s\right]=f(x) .
$$

Note, in particular, that $\tau$ is not assumed to be almost surely finite. We observe that

$$
\lim _{t \nearrow \infty} \mathrm{e}^{-\alpha(t \wedge \tau)} f(X(t \wedge \tau))=0
$$

on the set $\{\tau=\infty\}$, so $\mathbf{1}_{\{\tau<\infty\}}$ may be included in the limiting expression. The identity (2.2) holds for all $f \in C_{c}^{2}\left(x_{l}, x_{r}\right)$.

We now specialize (2.2) to the eigenfunctions $\phi$ and $\psi$ that solve (1.4). It is for this reason that we need to restrict the stopping times to the set $\mathcal{A}_{1}$. Let $\tau \in \mathcal{A}_{1}$ be chosen arbitrarily, and let $a_{\tau}$ and $b_{\tau}$ denote the values such that $x_{l}<a_{\tau} \leq X(t) \leq b_{\tau}<x_{r}$ for all $0 \leq t \leq \tau$. Select a mollifying function $\xi \in C_{c}^{2}\left(x_{l}, x_{r}\right)$ with $\xi(x)=1$ for $x \in\left[a_{\tau}, b_{\tau}\right]$. Taking $f=\xi \phi$ and $f=\xi \psi$ shows that (2.2) holds for the functions $\phi$ and $\psi$ with the added benefit that Af $-\alpha f \equiv 0$ on $\left[a_{\tau}, b_{\tau}\right]$ so the integral term is 0 .

The LP associated with the optimal stopping problem uses a discounted stopping distribution. Define the measure $\mu_{\tau}$ as

$$
\mu_{\tau}(G)=\mathrm{E}\left[\mathrm{e}^{-\alpha \tau} \mathbf{1}_{G}(X(\tau)) \mathbf{1}_{\{\tau<\infty\}}\right] \text { for all } G \in \mathcal{B}\left[x_{l}, x_{r}\right] .
$$

Observe that $\mu_{\tau}$ has total mass that is less than or equal to 1 . Taking the mollified $\phi$ and $\psi$ functions, the identity (2.2) can be expressed in terms of $\mu_{\tau}$ as

$$
\int \phi \mathrm{d} \mu_{\tau}=\phi(x) \text { and } \int \psi \mathrm{d} \mu_{\tau}=\psi(x)
$$

Note the dependence of this identity on the initial position $x$ of the diffusion $X$. Turning to the objective function (1.5), observe that it can also be expressed in terms of $\mu_{\tau}$ as

$$
J_{r}(\tau ; x)=\int g_{r} \mathrm{~d} \mu_{\tau} .
$$

We therefore observe that, for each stopping time $\tau \in \mathcal{A}_{1}$ and process $X$ satisfying (1.1), the corresponding measure $\mu_{\tau}$ satisfies (2.4) and the value $J_{r}(\tau ; x)$ is given by (2.5). Thus, the optimal stopping problem is imbedded in the LP

$$
\begin{cases}\text { Maximize } & \int g_{r} \mathrm{~d} \mu \\ \text { Subject to } & \int \phi \mathrm{d} \mu=\phi(x), \\ & \int \psi \mathrm{d} \mu=\psi(x), \\ & \int 1 \mathrm{~d} \mu \leq 1, \\ & \mu \text { is a nonnegative measure. }\end{cases}
$$


Denote the value of this LP by $V_{\mathrm{LP}}(x)$. It immediately follows that

$$
V_{1}(x) \leq V_{\mathrm{LP}}(x)
$$

We place the following additional assumption on the problem to ensure that $V_{\mathrm{LP}}(x)$ is finite.

Condition 2.1. For each $x \in\left(x_{l}, x_{r}\right)$,

$$
\sup _{x_{l} \leq y \leq x} \frac{g_{r}(y)}{\phi(y)}<\infty \text { and } \sup _{x \leq y \leq x_{r}} \frac{g_{r}(y)}{\psi(y)}<\infty .
$$

Since both $\phi$ and $\psi$ are bounded on compact subsets of $\left(x_{l}, x_{r}\right)$, Condition 2.1 requires that $g_{r}$ also be bounded on compact subsets of $\left(x_{l}, x_{r}\right)$. When $x_{l}$ or $x_{r}$ is an exit-not-entrance boundary point, $\phi\left(x_{l}+\right)<\infty$ or $\psi\left(x_{r}-\right)<\infty$, respectively, so Condition 2.1 implies that $g_{r}$ is bounded at such boundary points. In the case of natural or entrance-not-exit boundary points, $\phi(y)$ or $\psi(y)$ go to $\infty$ as $y$ approaches such a boundary point so $g_{r}$ may be unbounded near such points. We adopt Condition 2.1 since it captures these variations in a simple manner.

\subsection{Analysis of the hitting time of $\{a, b\}$}

The constraints of LP (2.6) can be used to determine the values corresponding to a simple stopping rule that plays a central role in the construction of the value function. In particular, we examine the reward obtained by a two-point hitting time of levels on both sides of $x$.

Fix $a$ and $b$ with $x_{l}<a \leq x \leq b<x_{r}$, and consider the stopping rule $\tau_{a, b}=\inf \{t \geq$ $0: X(t) \in\{a, b\}\}$, the first hitting time of either level $a$ or level $b$. Definition (2.3) implies that $\mu_{\tau_{a, b}}$ has $\{a, b\}$ for its support and hence (2.4) results in the system

$$
\phi(a) \mu_{\tau_{a, b}}(a)+\phi(b) \mu_{\tau_{a, b}}(b)=\phi(x), \quad \psi(a) \mu_{\tau_{a, b}}(a)+\psi(b) \mu_{\tau_{a, b}}(b)=\psi(x) .
$$

Two cases must be considered. When $a=x=b$, the equation involving $\phi$ reduces to $\phi(x) \mu_{\tau_{x}}(x)=\phi(x)$, and similarly for $\psi$ with solution $\mu_{\tau_{x}}(x)=1$. Now suppose that $a<b$. The solution of the system is then

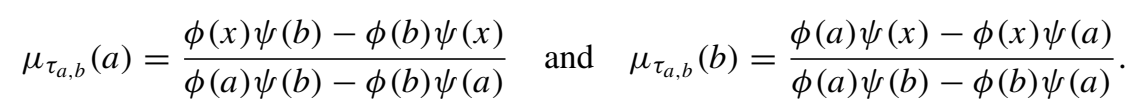

These masses are nonnegative since $\phi$ is decreasing and $\psi$ is increasing. It therefore follows that the reward associated with the stopping rule $\tau_{a, b}$ is

$$
J_{r}\left(\tau_{a, b} ; x\right)= \begin{cases}g_{r}(x), & a=b, \\ g_{r}(a) \frac{\phi(x) \psi(b)-\phi(b) \psi(x)}{\phi(a) \psi(b)-\phi(b) \psi(a)}+g_{r}(b) \frac{\phi(a) \psi(x)-\phi(x) \psi(a)}{\phi(a) \psi(b)-\phi(b) \psi(a)}, & a<b .\end{cases}
$$

Examining the expression for $J\left(\tau_{a, b} ; x\right)$ when $a<b$, we see that it simplifies to $g_{r}(x)$ when either $a=x<b$ or $a<x=b$.

Remark 2.1. We observe the following limiting results which agree with our intuition. For each $c \in\left(x_{l}, x_{r}\right)$, let $\tau_{c}=\inf \{t>0: X(t)=c\}$. When $x_{r}$ is either a natural boundary point or an entrance-not-exit boundary point, $\psi\left(x_{r}-\right)=\infty$. As a result, if we hold the left boundary point $a$ fixed and let $b \rightarrow x_{r}$, the expression for $\mu_{\tau_{a, b}}(a) \rightarrow \phi(x) / \phi(a)=\mu_{\tau_{a}}(a)$ (see [3, Section II.10, p. 18]) and $\mu_{\tau_{a, b}}(b) \rightarrow 0$. Hence, $J_{r}\left(\tau_{a, b} ; x\right)$ converges to

$$
J_{r}\left(\tau_{a} ; x\right)=\frac{\phi(x) g_{r}(a)}{\phi(a)} .
$$


Similarly, when $x_{l}$ is either a natural or entrance-not-exit boundary point, $\phi\left(x_{l}+\right)=\infty$, and holding $b$ fixed and letting $a \rightarrow x_{l}$ yields $\mu_{\tau_{a, b}}(a) \rightarrow 0$ and $\mu_{\tau_{a, b}}(b) \rightarrow \psi(x) / \psi(b)=\mu_{\tau_{b}}(b)$. Again, it follows that $J_{r}\left(\tau_{a, b} ; x\right)$ converges to

$$
J_{r}\left(\tau_{b} ; x\right)=\frac{\psi(x) g_{r}(b)}{\psi(b)} .
$$

A benefit of these observations is that our optimization of $J_{r}\left(\tau_{a, b} ; x\right)$ in Section 4 allows for one-sided stopping rules to be seen to be optimal.

Remark 2.2. Expression (2.9) for $J\left(\tau_{a, b} ; x\right)$ exhibits an interesting result when $g_{r}=c_{1} \phi+c_{2} \psi$ for any constants $c_{1}$ and $c_{2}$. This situation occurs when the running reward $r \equiv 0$ and the terminal reward is $g=c_{1} \phi+c_{2} \psi$. For each choice of stopping locations $a$ and $b$, by explicit computation, $J\left(\tau_{a, b} ; x\right)=c_{1} \phi(x)+c_{2} \psi(x)$ and, thus, every two-point stopping rule has the same value. Of course, this observation is merely a special case of (2.4).

Finally, observe that, for any choice of $a$ and $b$, with $x_{l}<a \leq x \leq b<x_{r}$, the reward obtained using stopping rule $\tau_{a, b}$ is no greater than the optimal reward:

$$
J_{r}\left(\tau_{a, b}, x\right) \leq V_{1}(x)
$$

\section{Dual LP and weak duality}

In this section we analyze the LP (2.6) using the dual LP and a further restricted LP. For the remainder of this section, fix $x \in\left(x_{l}, x_{r}\right)$ arbitrarily.

To develop a dual LP corresponding to (2.6), observe that, since there are three constraints, there will be three dual variables, which we denote by $c_{1}, c_{2}$, and $c_{3}$. The dual LP is

$$
\begin{cases}\text { Minimize } & c_{1} \phi(x)+c_{2} \psi(x)+c_{3} \\ \text { Subject to } & c_{1} \phi(y)+c_{2} \psi(y)+c_{3} 1(y) \geq g_{r}(y) \quad \text { for all } y \in\left[x_{l}, x_{r}\right] \\ & c_{1}, c_{2} \text { unrestricted } \\ & c_{3} \geq 0\end{cases}
$$

In this LP, the function 1 represents the constant function taking value 1 . Let $V_{\mathrm{DLP}}(x)$ denote the value of this dual LP.

Theorem 3.1. For $x \in\left(x_{l}, x_{r}\right)$,

$$
V_{\mathrm{DLP}}(x) \geq V_{\mathrm{LP}}(x) \text {. }
$$

Proof. Observe that Condition 2.1 implies the existence of feasible points for the dual LP (3.1), and taking $\mu=\delta_{\{x\}}$ shows that the feasible set for the LP (2.6) is nonempty. A standard weak duality argument therefore establishes the result.

Finally, we restrict the feasible set of the dual LP (3.1) by setting $c_{3}=0$. This results in a restricted dual LP

$$
\begin{cases}\text { Minimize } & c_{1} \phi(x)+c_{2} \psi(x) \\ \text { Subject to } & c_{1} \phi(y)+c_{2} \psi(y) \geq g_{r}(y) \text { for all } y \in\left[x_{l}, x_{r}\right] \\ & c_{1}, c_{2} \text { unrestricted. }\end{cases}
$$


Denote the value of (3.3) by $V_{\mathrm{RDLP}}(x)$. The above discussion implies that the feasible set of the dual LP (3.1) contains the feasible set of restricted dual LP (3.3), so

$$
V_{\mathrm{DLP}}(x) \leq V_{\mathrm{RDLP}}(x)
$$

Now look carefully at this restricted dual LP. The function $c_{1} \phi+c_{2} \psi$ satisfies the differential equation $A f-\alpha f=0$ and, to be feasible, is required to lie above the reward function. The goal of the LP is to pick the values $c_{1}$ and $c_{2}$ so as to minimize the objective function.

Combining the set of inequalities in (2.7), (2.12), (3.2), and (3.4) yields, for every $x_{l}<a \leq$ $x \leq b<x_{r}$ with $x \in\left(x_{l}, x_{r}\right)$,

$$
J_{r}\left(\tau_{a, b} ; x\right) \leq V_{1}(x) \leq V_{\mathrm{LP}}(x) \leq V_{\mathrm{DLP}}(x) \leq V_{\mathrm{RDLP}}(x) .
$$

At this point, it will be helpful for our further discussion to state clearly the weak duality result that will be exploited. Let $J_{r}^{*}(x)=\sup _{\left\{a, b: x_{l}<a \leq x \leq b<x_{r}\right\}} J_{r}\left(\tau_{a, b} ; x\right)$ denote the optimal value associated by restricting the stopping rules to the set $\left\{\tau_{a, b}: x_{l}<a \leq x \leq b<x_{r}\right\}$. Also, define $\widehat{A}$ to be the set of feasible $c_{1}, c_{2}$ for $(3.3)$ and let $\widehat{J}_{r}\left(c_{1}, c_{2} ; x\right)=c_{1} \phi(x)+c_{2} \psi(x)$. Then $V_{\mathrm{RDLP}}(x)=\inf _{c_{1}, c_{2} \in \widehat{\mathcal{A}}} \widehat{J}_{r}\left(c_{1}, c_{2} ; x\right)$.

Theorem 3.2. (Weak duality.) Let $a$ and $b$ satisfy $x_{l}<a \leq x \leq b<x_{r}$, and let $c_{1}$ and $c_{2}$ be feasible for (3.3). Then

$$
J_{r}\left(\tau_{a, b} ; x\right) \leq J_{r}^{*}(x) \leq V_{\mathrm{RDLP}}(x) \leq \widehat{J}_{r}\left(c_{1}, c_{2} ; x\right) .
$$

\section{Optimization of $J_{r}\left(\tau_{a, b} ; x\right)$ and strong duality}

We return to the examination of the reward $J_{r}\left(\tau_{a, b} ; x\right)$ associated with stopping at the first hitting time of $\{a, b\}$ and, in particular, we consider the optimization of this value over all possible choices of $a$ and $b$ with $x_{l} \leq a \leq x$ and $x \leq b \leq x_{r}$. Again, let $x \in\left(x_{l}, x_{r}\right)$ be fixed for this discussion.

\subsection{Existence of optimizers}

We begin by considering the situation in which $g_{r} \leq 0$ and with $x_{l}$ and $x_{r}$ being either natural boundaries or entrance-not-exit boundaries for the diffusion. This assumption means that $\tau_{x_{l}, x_{r}}=\infty$ almost surely and, moreover, $\phi\left(x_{l}+\right)=\psi\left(x_{r}-\right)=\infty$. In this setting, an optimal stopping rule is $\tau_{x_{l}, x_{r}}$ since $J_{r}\left(\tau_{x_{l}, x_{r}} ; x\right)=0$ and stopping at any finite locations $a$ and $b$ will result in a nonpositive expected reward.

For the rest of the optimization discussion, we assume that there is some $y \in\left(x_{l}, x_{r}\right)$ for which $g_{r}(y)>0$. We now impose conditions which imply that $J_{r}^{*}(x)$ is achieved by some points $a^{*} \in\left[x_{l}, x\right]$ and $b^{*} \in\left[x, x_{r}\right]$.

Condition 4.1. Assume that $g_{r}$ satisfies the following:

(a) $g_{r}$ is upper semicontinuous;

(b) if $x_{l}$ is either a natural or an entrance-not-exit boundary point, then

$$
\lim _{y \rightarrow x_{l}} \frac{g_{r}(y)}{\phi(y)}=0 ;
$$

and 
(c) if $x_{r}$ is either a natural or an entrance-not-exit boundary point, then

$$
\lim _{y \rightarrow x_{r}} \frac{g_{r}(y)}{\psi(y)}=0
$$

Since $f_{r}$ is continuous, Condition 4.1(a) requires $g$ to be upper semicontinuous.

Theorem 4.1. Under Conditions 2.1 and 4.1, for each $x \in\left(x_{l}, x_{r}\right)$, there exist values $a^{*}=$ $a^{*}(x) \in\left[x_{l}, x\right]$ and $b^{*}=b^{*}(x) \in\left[x, x_{r}\right]$ such that $J_{r}\left(\tau_{a^{*}, b^{*}} ; x\right)=J_{r}^{*}(x)$.

Proof. When $J_{r}^{*}(x)=g_{r}(x)$, the choice of $a^{*}=x=b^{*}$ satisfies the claim. So assume that $J_{r}^{*}(x)>g_{r}(x)$. Let $\left\{\left(a_{n}, b_{n}\right): n \in \mathbb{N}\right\}$ be a sequence with $a_{n}<x$ and $b_{n}>x$ for all $n \in \mathbb{N}$ such that $J_{r}\left(\tau_{a_{n}, b_{n}} ; x\right) \rightarrow J_{r}^{*}(x)$ as $n \rightarrow \infty$.

Compactify the interval $\left[x_{l}, x_{r}\right]$ when $x_{l}$ and/or $x_{r}$ are either natural or entrance-not-exit boundary points. It then follows that there exists a subsequence $\left\{n_{k}\right\}$ and values $a^{*}$ and $b^{*}$ such that $a_{n_{k}} \rightarrow a^{*}$ and $b_{n_{k}} \rightarrow b^{*}$. To simplify notation, assume that the original sequence has the properties of this subsequence. The following set of relations then holds:

$$
\begin{aligned}
J_{r}^{*}(x) & =\lim _{n \rightarrow \infty} J_{r}\left(\tau_{a_{n}, b_{n}} ; x\right) \\
& =\lim _{n \rightarrow \infty}\left(g_{r}\left(a_{n}\right) \frac{\phi(x) \psi\left(b_{n}\right)-\phi\left(b_{n}\right) \psi(x)}{\phi\left(a_{n}\right) \psi\left(b_{n}\right)-\phi\left(b_{n}\right) \psi\left(a_{n}\right)}+g_{r}\left(b_{n}\right) \frac{\phi\left(a_{n}\right) \psi(x)-\phi(x) \psi\left(a_{n}\right)}{\phi\left(a_{n}\right) \psi\left(b_{n}\right)-\phi\left(b_{n}\right) \psi\left(a_{n}\right)}\right) \\
& \leq g_{r}\left(a^{*}\right) \frac{\phi(x) \psi\left(b^{*}\right)-\phi\left(b^{*}\right) \psi(x)}{\phi\left(a^{*}\right) \psi\left(b^{*}\right)-\phi\left(b^{*}\right) \psi\left(a^{*}\right)}+g_{r}\left(b^{*}\right) \frac{\phi\left(a^{*}\right) \psi(x)-\phi(x) \psi\left(a^{*}\right)}{\phi\left(a^{*}\right) \psi\left(b^{*}\right)-\phi\left(b^{*}\right) \psi\left(a^{*}\right)} \\
& =J_{r}\left(\tau_{a^{*}, b^{*}} ; x\right) \\
& \leq J_{r}^{*}(x) ;
\end{aligned}
$$

the first inequality follows since the continuity of $\phi$ and $\psi$ imply the convergence of the fractions and $g_{r}$ is upper semicontinuous. Thus, equality holds throughout these relations and $\tau_{a^{*}, b^{*}}$ is an optimal stopping time.

To be precise, should $a^{*}=x_{l}$ with $x_{l}$ being a natural or an entrance-not-exit boundary point and $b^{*}$ be an interior point of the interval $\left[x_{l}, x_{r}\right]$, the limiting expression is $\left(\psi(x) / \psi\left(b^{*}\right)\right)$ $g_{r}\left(b^{*}\right)=J_{r}\left(\tau_{b^{*}} ; x\right)$ and $\tau_{b^{*}}$ is optimal. A similar comment applies to the case of $b^{*}=x_{r}$ with $a^{*} \in\left(x_{l}, x_{r}\right)$, yielding $\tau_{a^{*}}$ as an optimal stopping time.

The case in which $a^{*}=x_{l}$ and $b^{*}=x_{r}$ with both boundary points being either natural or entrance-not-exit does not arise. For if it did, Condition 4.1(b) and (c) imply that the coefficients of $g_{r}\left(a^{*}\right)$ and $g_{r}\left(b^{*}\right)$ would be 0 , corresponding to $\tau_{a^{*}, b^{*}}=\infty$ almost surely, and, hence, $J_{r}\left(\tau_{a^{*}, b^{*}} ; x\right)=0$. But there exists some $y \in\left(x_{l}, x_{r}\right)$ with $g_{r}(y)>0$. The stopping time $\tau_{y}$ which stops the process when it first hits $\{y\}$ will have a strictly positive value for $J_{r}\left(\tau_{y} ; x\right)$, contradicting $0=\lim _{n \rightarrow \infty} J_{r}\left(\tau_{a_{n}, b_{n}} ; x\right)=J_{r}^{*}(x)$.

Remark 4.1. The above proof only uses upper semicontinuity of $g_{r}$ at the optimizing points $a^{*}$ and $b^{*}$. We would therefore be able to relax the upper semicontinuity assumption on $g_{r}$ so that it is required to only hold at the optimizers.

\subsection{Strong duality}

At this point an observation is very helpful in preparation for the proof of the strong duality theorem. To this point we have been considering a single initial point $x$ and the LPs related to it. The value function $V_{r}$ is a function of the initial position and we will prove that the values of the family of LPs parameterized by $x$ give $V_{r}$. It is thus beneficial to consider more than a 
single initial value at a time. For instance, should $x$ be an initial value such that $a^{*}<x<b^{*}$, then the optimization of $J\left(\tau_{a, b} ; \tilde{x}\right)$ for every other initial value $\tilde{x} \in\left(a^{*}, b^{*}\right)$ implies that $a^{*}$ and $b^{*}$ are also optimal for $\tilde{x}$. The nondegenerate interval $\left(a^{*}, b^{*}\right)$ is thus seen to be part of the continuation region $\mathcal{C}$ described in the introduction in which it is optimal to allow the process $X$ to continue without stopping. Let $\mathcal{C}=\cup\left\{x \in\left(x_{l}, x_{r}\right): a^{*}(x)<x<b^{*}(x)\right\}$ be the continuation region. The set $\delta=\left[x_{l}, x_{r}\right] \cap \mathcal{C}^{\mathrm{c}}$ is the stopping region. Let $\overline{\mathrm{C}}$ denote the closure of $\mathcal{C}$, and let $\delta^{\circ}$ denote the interior of $\delta$.

The following proposition identifies a condition under which $x$ is an element of $\overline{\mathcal{C}}$.

Proposition 4.1. If $\limsup _{y \rightarrow x} g_{r}(y)<g_{r}(x)$ then $x \in \overline{\mathcal{C}}$.

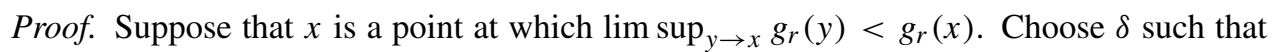
$0<\delta<g_{r}(x)-\lim \sup _{y \rightarrow x} g_{r}(y)$. Let $y_{1}<x$ be fixed, and consider the stopping rule $\tau_{y_{1}, x}$ when the initial value is $y$ with $y_{1}<y<x$. The value associated with this rule is

$$
J_{r}\left(\tau_{y_{1}, x} ; y\right)=\frac{\phi(y) \psi(x)-\phi(x) \psi(y)}{\phi\left(y_{1}\right) \psi(x)-\phi(x) \psi\left(y_{1}\right)} g_{r}\left(y_{1}\right)+\frac{\phi\left(y_{1}\right) \psi(y)-\phi(y) \psi\left(y_{1}\right)}{\phi\left(y_{1}\right) \psi(x)-\phi(x) \psi\left(y_{1}\right)} g_{r}(x) .
$$

Observe that the coefficient of $g_{r}\left(y_{1}\right)$ converges to 0 as $y$ converges to $x$ and, similarly, the coefficient of $g_{r}(x)$ converges to 1 . Select $y_{2}<x$ such that, for all $y_{2}<y<x, g_{r}(y)<$ $g_{r}(x)-\delta$,

$\left|\frac{\phi(y) \psi(x)-\phi(x) \psi(y)}{\phi\left(y_{1}\right) \psi(x)-\phi(x) \psi\left(y_{1}\right)} g_{r}\left(y_{1}\right)\right|<\frac{\delta}{3} \quad$ and $\quad \frac{\phi\left(y_{1}\right) \psi(y)-\phi(y) \psi\left(y_{1}\right)}{\phi\left(y_{1}\right) \psi(x)-\phi(x) \psi\left(y_{1}\right)} g_{r}(x)>g_{r}(x)-\frac{\delta}{3}$.

Then, for all $y_{2}<y<x, J_{r}\left(\tau_{y_{1}, x} ; y\right)>g_{r}(y)$ and $y \in \mathcal{C}$.

Having established the existence of optimizers $a^{*}=a^{*}(x)$ and $b^{*}=b^{*}(x)$ for the optimal stopping problem restricted to two-point stopping rules, the goal is to prove the optimality of $\tau_{a^{*}, b^{*}}$ for the general stopping problem. Our approach will be to obtain coefficients $c_{1}^{*}$ and $c_{2}^{*}$ that are feasible for the restricted dual LP with $\widehat{J}\left(c_{1}^{*}, c_{2}^{*} ; x\right)=J\left(\tau_{a^{*}, b^{*}} ; x\right)$ and, thus, equality will hold throughout (3.5) and (3.6). To achieve this result, we must further restrict the class of reward functions.

Condition 4.2. For each $x \in\left(x_{l}, x_{r}\right)$ for which $\lim _{\sup _{y \rightarrow x}} g(y)=g(x)$,

(a) we have

$$
-\infty<\liminf _{y \nearrow x} \frac{g(y)-g(x)}{y-x}<\infty \text { and } \quad-\infty<\limsup _{z \searrow x} \frac{g(z)-g(x)}{z-x}<\infty ;
$$

and

(b) if $x$ is a point where

$$
\liminf _{y \nearrow x} \frac{g(y)-g(x)}{y-x} \neq \limsup _{z \searrow x} \frac{g(z)-g(x)}{z-x}
$$

then there exists a sequence $\left\{y_{n}<x: n \in \mathbb{N}\right\}$, with $y_{n} \rightarrow x$ and

$$
\frac{g\left(y_{n}\right)-g(x)}{y_{n}-x} \rightarrow \liminf _{y \nearrow x} \frac{g(y)-g(x)}{y-x} \text { as } n \rightarrow \infty
$$


such that, for each $n$,

$$
\liminf _{y \nearrow y_{n}} \frac{g(y)-g\left(y_{n}\right)}{y-y_{n}}=\limsup _{z \searrow y_{n}} \frac{g(z)-g\left(y_{n}\right)}{z-y_{n}},
$$

or there exists a sequence $\left\{z_{n}>x: n \in \mathbb{N}\right\}$ with $\lim _{n \rightarrow \infty} z_{n}=x$ and

$$
\lim _{n \rightarrow \infty} \frac{g\left(z_{n}\right)-g(x)}{z_{n}-x}=\limsup _{z \searrow x} \frac{g(z)-g(x)}{z-x}
$$

such that (4.1) is satisfied for each $n$ when $z_{n}$ replaces $y_{n}$.

Observe that Condition 4.2(b) is satisfied, for example, when $g$ is $C^{1}$ in either a left neighborhood or a right neighborhood of $x$ with the left-hand derivative or right-hand derivative of $g$, respectively, existing at $x$.

Since $f_{r} \in C^{2}\left[x_{l}, x_{r}\right]$, the function $g_{r}=f_{r}+g$ inherits the properties in Condition 4.2 from $g$. The proof of our main result uses these properties of $g_{r}$.

Theorem 4.2. (Strong duality.) Under Conditions 2.1, 4.1, and 4.2 on g or $g_{r}$, for $x \in\left(x_{l}, x_{r}\right)$, there exist stopping locations $a^{*} \in\left[x_{l}, x\right]$ and $b^{*} \in\left[x, x_{r}\right]$ and coefficients $\left(c_{1}^{*}, c_{2}^{*}\right) \in \widehat{\mathcal{A}}$ such that

$$
J_{r}\left(\tau_{a^{*}, b^{*}} ; x\right)=J_{r}^{*}(x)=V_{1}(x)=V_{\mathrm{RDLP}}(x)=\widehat{J}_{r}\left(c_{1}^{*}, c_{2}^{*} ; x\right) .
$$

Proof. The existence of $a^{*}$ and $b^{*}$ such that $J_{r}\left(\tau_{a^{*}, b^{*}} ; x\right)=J_{r}^{*}(x)$ follows from Theorem 4.1. Note in (2.9) that, when $a=x$ or $b=x, J_{r}\left(\tau_{x, b} ; x\right)=g_{r}(x)$ so

$$
J_{r}^{*}(x)=\sup _{\left\{a, b: x_{l} \leq a \leq x \leq b \leq x_{r}\right\}} J_{r}\left(\tau_{a, b} ; x\right) \geq g_{r}(x) .
$$

It is necessary to consider different cases for the initial value $x$. Before doing so, however, we examine the value associated with a two-point hitting rule and establish some notation.

Observe that the expression for $J_{r}\left(\tau_{a, b} ; x\right)$ in (2.9) with $a<x<b$ can be rewritten as

$$
J_{r}\left(\tau_{a, b} ; x\right)=\frac{g_{r}(a) \psi(b)-g_{r}(b) \psi(a)}{\phi(a) \psi(b)-\phi(b) \psi(a)} \phi(x)+\frac{g_{r}(b) \phi(a)-g_{r}(a) \phi(b)}{\phi(a) \psi(b)-\phi(b) \psi(a)} \psi(x) .
$$

Define the coefficients $c_{1}$ and $c_{2}$ by

$$
c_{1}(a, b)=\frac{g_{r}(a) \psi(b)-g_{r}(b) \psi(a)}{\phi(a) \psi(b)-\phi(b) \psi(a)} \quad \text { and } \quad c_{2}(a, b)=\frac{g_{r}(b) \phi(a)-g_{r}(a) \phi(b)}{\phi(a) \psi(b)-\phi(b) \psi(a)} .
$$

Now, let

$$
J_{a, b}(y)=c_{1}(a, b) \phi(y)+c_{2}(a, b) \psi(y) \text { for } y \in\left[x_{l}, x_{r}\right] ;
$$

that is, we define the function $J_{a, b}$ on $\left[x_{l}, x_{r}\right]$ to have the form of $J_{r}\left(\tau_{a, b} ; x\right)$ in (4.2), but do not require the independent variable to lie in the interval $(a, b)$.

Case (a). Suppose that $x \in \mathcal{C}$. Then there are points $a^{*}$ and $b^{*}$ such that

$$
a^{*}=a^{*}(x)<x<b^{*}(x)=b^{*} .
$$


We claim that $J_{a^{*}, b^{*}} \geq g_{r}$. To verify this claim, consider first $a=y<x$ and $b=b^{*}$. Then $J_{r}\left(\tau_{y, b^{*}} ; x\right) \leq J_{r}\left(\tau_{a^{*}, b^{*}} ; x\right)$ implies that

$$
c_{1}\left(y, b^{*}\right) \phi(x)+c_{2}\left(y, b^{*}\right) \psi(x) \leq c_{1}\left(a^{*}, b^{*}\right) \phi(x)+c_{2}\left(a^{*}, b^{*}\right) \psi(x) .
$$

Using the definitions of $c_{1}$ and $c_{2}$ in (4.3) and rewriting the expressions as in (2.9), we have

$$
\begin{aligned}
& \frac{\phi(x) \psi\left(b^{*}\right)-\phi\left(b^{*}\right) \psi(x)}{\phi(y) \psi\left(b^{*}\right)-\phi\left(b^{*}\right) \psi(y)} g_{r}(y)+\frac{\phi(y) \psi(x)-\phi(x) \psi(y)}{\phi(y) \psi\left(b^{*}\right)-\phi\left(b^{*}\right) \psi(y)} g_{r}\left(b^{*}\right) \\
& \quad \leq \frac{\phi(x) \psi\left(b^{*}\right)-\phi\left(b^{*}\right) \psi(x)}{\phi\left(a^{*}\right) \psi\left(b^{*}\right)-\phi\left(b^{*}\right) \psi\left(a^{*}\right)} g_{r}\left(a^{*}\right)+\frac{\phi\left(a^{*}\right) \psi(x)-\phi(x) \psi\left(a^{*}\right)}{\phi\left(a^{*}\right) \psi\left(b^{*}\right)-\phi\left(b^{*}\right) \psi\left(a^{*}\right)} g_{r}\left(b^{*}\right) .
\end{aligned}
$$

Isolating $g_{r}(y)$ on the left-hand-side results in

$$
\begin{aligned}
g_{r}(y) \leq & \frac{\phi(y) \psi\left(b^{*}\right)-\phi\left(b^{*}\right) \psi(y)}{\phi\left(a^{*}\right) \psi\left(b^{*}\right)-\phi\left(b^{*}\right) \psi\left(a^{*}\right)} g_{r}\left(a^{*}\right) \\
& +\left(\frac{\phi(y) \psi\left(b^{*}\right)-\phi\left(b^{*}\right) \psi(y)}{\phi(x) \psi\left(b^{*}\right)-\phi\left(b^{*}\right) \psi(x)}\right) \\
& \quad \times\left[\frac{\phi\left(a^{*}\right) \psi(x)-\phi(x) \psi\left(a^{*}\right)}{\phi\left(a^{*}\right) \psi\left(b^{*}\right)-\phi\left(b^{*}\right) \psi\left(a^{*}\right)}-\frac{\phi(y) \psi(x)-\phi(x) \psi(y)}{\phi(y) \psi\left(b^{*}\right)-\phi\left(b^{*}\right) \psi(y)}\right] g_{r}\left(b^{*}\right) \\
= & \frac{\phi(y) \psi\left(b^{*}\right)-\phi\left(b^{*}\right) \psi(y)}{\phi\left(a^{*}\right) \psi\left(b^{*}\right)-\phi\left(b^{*}\right) \psi\left(a^{*}\right)} g_{r}\left(a^{*}\right)+\frac{\phi\left(a^{*}\right) \psi(y)-\phi(y) \psi\left(a^{*}\right)}{\phi\left(a^{*}\right) \psi\left(b^{*}\right)-\phi\left(b^{*}\right) \psi\left(a^{*}\right)} g_{r}\left(b^{*}\right) \\
= & c_{1}\left(a^{*}, b^{*}\right) \phi(y)+c_{2}\left(a^{*}, b^{*}\right) \psi(y) \\
= & J_{a^{*}, b^{*}}(y) .
\end{aligned}
$$

Using a similar computation with $a=a^{*}$ and $b=y>x$ establishes the claim. We therefore see that this choice of $c_{1}^{*}=c_{1}\left(a^{*}, b^{*}\right)$ and $c_{2}^{*}=c_{2}\left(a^{*}, b^{*}\right)$ is feasible for the restricted dual LP (3.3) and $\left(a^{*}, b^{*}\right)$ is optimal for the problem of maximizing $J_{r}\left(\tau_{a, b} ; x\right)$ over $a$ and $b$, and, moreover, by the definition of $c_{1}^{*}$ and $c_{2}^{*}, J_{r}\left(\tau_{a^{*}, b^{*}} ; x\right)=\widehat{J}_{r}\left(c_{1}^{*}, c_{2}^{*} ; x\right)$.

Case (b). Suppose that $x \in \partial \mathcal{C}$, the boundary of $\mathcal{C}$. There are two cases to consider.

(i) Suppose that $x$ is a point at which $\lim _{\sup _{y \rightarrow x}} g_{r}(y)<g_{r}(x)$. Since $x \notin \mathcal{C}$, the proof of Proposition 4.1 shows that, for $y$ sufficiently close to $x$, with $y<x, y \in \mathcal{C}$ and $b^{*}(y)=x$. Since $a^{*}(y)<b^{*}(y)=x$, the result of case (a) applies so that fixing $y<x$ sufficiently close to $x$ and defining $c_{1}^{*}=c_{1}\left(a^{*}(y), x\right)$ and $c_{2}^{*}=c_{2}\left(a^{*}(y), x\right)$, the function $c_{1}^{*} \phi+c_{2}^{*} \psi$ majorizes $g_{r}$ with equality holding at $x$.

(ii) Now suppose that $x \in \partial \mathcal{C}$ with $\limsup _{y \rightarrow x} g_{r}(y)=g_{r}(x)$. Then there exists a sequence $\left\{x_{n} \in \mathcal{C}: n \in \mathbb{N}\right\}$ such that $\lim _{n \rightarrow \infty} x_{n} \rightarrow x$. Without loss of generality, assume that $x_{n} \searrow x$. By considering a subsequence, if necessary, we may assume that

$$
\frac{g_{r}\left(x_{n}\right)-g_{r}(x)}{x_{n}-x} \rightarrow \limsup _{n \rightarrow \infty} \frac{g_{r}\left(x_{n}\right)-g_{r}(x)}{x_{n}-x}=: m .
$$

Since each $x_{n} \in \mathcal{C}, a_{n}^{*}:=a^{*}\left(x_{n}\right)<b^{*}\left(x_{n}\right)=: b_{n}^{*}$. Observe that $x \leq a_{n}^{*}<x_{n}$ so, as $n \rightarrow \infty$, $a_{n}^{*} \searrow x$. Should $b_{n}^{*}$ converge to some value $b^{*} \in\left(x, x_{r}\right]$, the proof of case (a) applies. So assume that $b_{n}^{*} \searrow x$ as $n \rightarrow \infty$. Define $c_{1}^{n}=c_{1}\left(a_{n}^{*}, b_{n}^{*}\right)$ and $c_{2}^{n}=c_{2}\left(a_{n}^{*}, b_{n}^{*}\right)$. It then follows that $c_{1}^{n} \phi+c_{2}^{n} \psi$ majorizes $g_{r}$ with equality holding at $x_{n}$. We investigate the limit of $c_{1}^{n}$ as 
$n \rightarrow \infty$. Observe that

$$
\begin{aligned}
c_{1}\left(a_{n}^{*}, b_{n}^{*}\right)= & \frac{g_{r}\left(a_{n}^{*}\right) \psi\left(b_{n}^{*}\right)-g_{r}\left(b_{n}^{*}\right) \psi\left(a_{n}^{*}\right)}{\phi\left(a_{n}^{*}\right) \psi\left(b_{n}^{*}\right)-\phi\left(b_{n}^{*}\right) \psi\left(a_{n}^{*}\right)} \\
= & \frac{g_{r}\left(a_{n}^{*}\right) \psi\left(b_{n}^{*}\right)-g_{r}\left(a_{n}^{*}\right) \psi\left(a_{n}^{*}\right)+g_{r}\left(a_{n}^{*}\right) \psi\left(a_{n}^{*}\right)-g_{r}\left(b_{n}^{*}\right) \psi\left(a_{n}^{*}\right)}{\phi\left(a_{n}^{*}\right) \psi\left(b_{n}^{*}\right)-\phi\left(a_{n}^{*}\right) \psi\left(a_{n}^{*}\right)+\phi\left(a_{n}^{*}\right) \psi\left(a_{n}^{*}\right)-\phi\left(b_{n}^{*}\right) \psi\left(a_{n}^{*}\right)} \\
= & \left(g_{r}\left(a_{n}^{*}\right) \frac{\psi\left(b_{n}^{*}\right)-\psi\left(a_{n}^{*}\right)}{b_{n}^{*}-a_{n}^{*}}-\frac{g_{r}\left(b_{n}^{*}\right)-g_{r}\left(a_{n}^{*}\right)}{b_{n}^{*}-a_{n}^{*}} \psi\left(a_{n}^{*}\right)\right) \\
& \times\left(\phi\left(a_{n}^{*}\right) \frac{\psi\left(b_{n}^{*}\right)-\psi\left(a_{n}^{*}\right)}{b_{n}^{*}-a_{n}^{*}}-\frac{\phi\left(b_{n}^{*}\right)-\phi\left(a_{n}^{*}\right)}{b_{n}^{*}-a_{n}^{*}} \psi\left(a_{n}^{*}\right)\right)^{-1} .
\end{aligned}
$$

Letting $n \rightarrow \infty$, we see that

$$
c_{1}^{n} \rightarrow \frac{g_{r}(x) \psi^{\prime}(x)-m \psi(x)}{\phi(x) \psi^{\prime}(x)-\phi^{\prime}(x) \psi(x)}=: c_{1}^{+} .
$$

A similar analysis shows that, as $n \rightarrow \infty$,

$$
c_{2}^{n} \rightarrow \frac{m \phi(x)-g_{r}(x) \phi^{\prime}(x)}{\phi(x) \psi^{\prime}(x)-\phi^{\prime}(x) \psi(x)}=: c_{2}^{+}
$$

and, thus, $c_{1}^{n} \phi+c_{2}^{n} \psi$ converges to $c_{1}^{+} \phi+c_{2}^{+} \psi$. Therefore, $c_{1}^{+} \phi+c_{2}^{+} \psi$ majorizes $g_{r}$ and, moreover, $\left[c_{1}^{+} \phi+c_{2}^{+} \psi\right](x)=g_{r}(x)$.

Case (c). Suppose that $x \in 8^{\circ}$. Then $a^{*}(x)=x=b^{*}(x)$. In this case, $c_{1}^{*}=c_{1}(x, x)$ and $c_{2}^{*}=c_{2}(x, x)$ are not defined and we need a different argument.

(i) To begin, consider the situation in which

$$
\liminf _{y \nearrow x} \frac{g_{r}(y)-g_{r}(x)}{y-x}=\limsup _{z \searrow x} \frac{g_{r}(z)-g_{r}(x)}{z-x}=: m .
$$

We seek constants $c_{1}$ and $c_{2}$ such that the function $c_{1} \phi+c_{2} \psi$ majorizes $g_{r}$ and equality holds at the initial value $x$. Consider the system of equations

$$
c_{1} \phi(x)+c_{2} \psi(x)=g_{r}(x), \quad c_{1} \phi^{\prime}(x)+c_{2} \psi^{\prime}(x)=m .
$$

The solution to this system is

$$
c_{1}=\frac{g_{r}(x) \psi^{\prime}(x)-m \psi(x)}{\phi(x) \psi^{\prime}(x)-\phi^{\prime}(x) \psi(x)} \quad \text { and } \quad c_{2}=\frac{m \phi(x)-g_{r}(x) \phi^{\prime}(x)}{\phi(x) \psi^{\prime}(x)-\phi^{\prime}(x) \psi(x)} .
$$

We claim the function $c_{1} \phi+c_{2} \psi$ majorizes $g_{r}$.

To see this, let $\varepsilon>0$ be chosen arbitrarily and let $y<x$ be arbitrary, $z>x$ will be specified later. The optimality of $\tau_{x}$ implies that $J_{r}\left(\tau_{y, z} ; x\right) \leq J_{r}\left(\tau_{x} ; x\right)=g_{r}(x)$. Writing $J_{r}\left(\tau_{y, z} ; x\right)$ as in (2.9) and isolating $g_{r}(y)$ leads to the inequality

$$
\begin{aligned}
g_{r}(y) & \leq \frac{\phi(y) \psi(z)-\phi(z) \psi(y)}{\phi(x) \psi(z)-\phi(z) \psi(x)} g_{r}(x)-\frac{\phi(y) \psi(x)-\phi(x) \psi(y)}{\phi(x) \psi(z)-\phi(z) \psi(x)} g_{r}(z) \\
& =\frac{g_{r}(x) \psi(z)-g_{r}(z) \psi(x)}{\phi(x) \psi(z)-\phi(z) \psi(x)} \phi(y)+\frac{g_{r}(z) \phi(x)-g_{r}(x) \phi(z)}{\phi(x) \psi(z)-\phi(z) \psi(x)} \psi(y) .
\end{aligned}
$$


Now, as in case (b)(ii), examine the coefficient of $\phi(y)$. We have

$$
\begin{aligned}
\frac{g_{r}(x) \psi(z)-g_{r}(z) \psi(x)}{\phi(x) \psi(z)-\phi(z) \psi(x)}= & \frac{g_{r}(x) \psi(z)-g_{r}(x) \psi(x)+g_{r}(x) \psi(x)-g_{r}(z) \psi(x)}{\phi(x) \psi(z)-\phi(x) \psi(x)+\phi(x) \psi(x)-\phi(z) \psi(x)} \\
= & \left(g_{r}(x) \frac{\psi(z)-\psi(x)}{z-x}-\frac{g_{r}(z)-g_{r}(x)}{z-x} \psi(x)\right) \\
& \times\left(\phi(x) \frac{\psi(z)-\psi(x)}{z-x}-\frac{\phi(z)-\phi(x)}{z-x} \psi(x)\right)^{-1}
\end{aligned}
$$

and, thus, letting $\left\{z_{n}>z: n \in \mathbb{N}\right\}$ be a sequence with $z_{n} \rightarrow x$ such that $\left(g_{r}\left(z_{n}\right)-g_{r}(x)\right) /\left(z_{n}-\right.$ $x) \rightarrow m$, it follows that

$$
\lim _{n \rightarrow \infty} \frac{g_{r}(x) \psi\left(z_{n}\right)-g_{r}\left(z_{n}\right) \psi(x)}{\phi(x) \psi\left(z_{n}\right)-\phi\left(z_{n}\right) \psi(x)}=\frac{g_{r}(x) \psi^{\prime}(x)-m \psi(x)}{\phi(x) \psi^{\prime}(x)-\phi^{\prime}(x) \psi(x)} .
$$

Arguing similarly with the coefficient of $\psi(y)$ yields

$$
\lim _{n \rightarrow \infty} \frac{g_{r}\left(z_{n}\right) \phi(x)-g_{r}(x) \phi\left(z_{n}\right)}{\phi(x) \psi\left(z_{n}\right)-\phi\left(z_{n}\right) \psi(x)}=\frac{m \phi(x)-g_{r}(x) \phi^{\prime}(x)}{\phi(x) \psi^{\prime}(x)-\phi^{\prime}(x) \psi(x)} .
$$

Recalling that $y$ is fixed, let $N \in \mathbb{N}$ be such that, for all $n \geq N$,

$$
\left|\frac{g_{r}(x) \psi\left(z_{n}\right)-g_{r}\left(z_{n}\right) \psi(x)}{\phi(x) \psi\left(z_{n}\right)-\phi\left(z_{n}\right) \psi(x)}-\frac{g_{r}(x) \psi^{\prime}(x)-m \psi(x)}{\phi(x) \psi^{\prime}(x)-\phi^{\prime}(x) \psi(x)}\right|<\frac{\varepsilon}{\phi(y)+\psi(y)}
$$

and

$$
\left|\frac{g_{r}\left(z_{n}\right) \phi(x)-g_{r}(x) \phi\left(z_{n}\right)}{\phi(x) \psi\left(z_{n}\right)-\phi\left(z_{n}\right) \psi(x)}-\frac{m \phi(x)-g_{r}(x) \phi^{\prime}(x)}{\phi(x) \psi^{\prime}(x)-\phi^{\prime}(x) \psi(x)}\right|<\frac{\varepsilon}{\phi(y)+\psi(y)} .
$$

Using the estimates in (4.7) and (4.8) in (4.6) yields

$$
\begin{aligned}
g_{r}(y) & \leq \frac{g_{r}(x) \psi^{\prime}(x)-m \psi(x)}{\phi(x) \psi^{\prime}(x)-\phi^{\prime}(x) \psi(x)} \phi(y)+\frac{m \phi(x)-g_{r}(x) \phi^{\prime}(x)}{\phi(x) \psi^{\prime}(x)-\phi^{\prime}(x) \psi(x)} \psi(y)+\varepsilon \\
& =c_{1} \phi(y)+c_{2} \psi(y)+\varepsilon .
\end{aligned}
$$

Since $\varepsilon>0$ is arbitrary, the claim holds for all $y<x$.

A similar argument with $z>x$ chosen arbitrarily and $y_{n}<x$ chosen in a similar approximating sequence close enough to $x$ establishes the relation for $z>x$. Thus, when

$$
\liminf _{y \nearrow x} \frac{g_{r}(y)-g_{r}(x)}{y-x}=\limsup _{z \searrow x} \frac{g_{r}(z)-g_{r}(x)}{z-x}=m
$$

at $x$ and $a^{*}=x=b^{*}$, we see that defining $c_{1}$ and $c_{2}$ as in (4.5) produces the function $c_{1} \phi+c_{2} \psi$ which majorizes $g_{r}$ with $\left[c_{1} \phi+c_{2} \psi\right](x)=g_{r}(x)$ and, hence, $\tau_{x}=0$ is optimal.

(ii) Suppose that $g_{r}$ satisfies Condition 4.2(b) at $x$ with a ' $>$ ' inequality and, for simplicity, assume that there is a left-approximating sequence $\left\{y_{n}<x: n \in \mathbb{N}\right\}$ and let $m_{n}:=$ $\liminf _{y} y_{n}\left(g_{r}(y)-g_{r}\left(y_{n}\right)\right) /\left(y-y_{n}\right)$. A similar argument will apply when a right-approximating sequence $\left\{z_{n}>x: n \in \mathbb{N}\right\}$ exists.

Let $m:=\liminf _{y}{ }_{x}\left(g_{r}(y)-g_{r}(x)\right) /(y-x)$, and define the coefficients $c_{1}^{-}$and $c_{2}^{-}$to be the solutions of the system (4.4). We claim that the function $c_{1}^{-} \phi+c_{2}^{-} \psi$ majorizes $g_{r}$ and 
satisfies $\left[c_{1}^{-} \phi+c_{2}^{-} \psi\right](x)=g_{r}(x)$. The latter condition follows immediately from the first equation in the system, so we only need to show that $c_{1}^{-} \phi+c_{2}^{-} \psi$ majorizes $g_{r}$.

Since $x \in 8^{\circ}$, there is some $\delta$ such that, for all $\tilde{x} \in(x-\delta, x), a^{*}(\tilde{x})=\tilde{x}=b^{*}(\tilde{x})$. For $y_{n} \in(x-\delta, x)$, define $c_{1}^{n}$ and $c_{2}^{n}$ as in (4.5) with $m_{n}$ replacing $m$. Case (b)(i) then implies that $c_{1}^{n} \phi+c_{2}^{n} \psi$ majorizes $g_{r}$ and $\left[c_{1}^{n} \phi+c_{2}^{n} \psi\right]\left(y_{n}\right)=g_{r}\left(y_{n}\right)$. Since $\left(g_{r}\left(y_{n}\right)-g_{r}(x)\right) /\left(y_{n}-x\right) \rightarrow m$ as $n \rightarrow \infty$, it follows that $g_{r}\left(y_{n}\right) \rightarrow g_{r}(x)$. Letting $n \rightarrow \infty$, the continuity of the derivatives of $\phi$ and $\psi$ and the existence of the finite limit $m$ of $\left(g_{r}\left(y_{n}\right)-g_{r}(x)\right) /\left(y_{n}-x\right)$ implies that $c_{1}^{n} \rightarrow c_{1}^{-}$and $c_{2}^{n} \rightarrow c_{2}^{-}$, and, hence, $c_{1}^{n} \phi+c_{2}^{n} \psi \rightarrow c_{1}^{-} \phi+c_{2}^{-} \psi$. Therefore, $c_{1}^{-} \phi+c_{2}^{-} \psi$ majorizes $g_{r}$.

Observe that, when $g_{r}$ satisfies both approximating sequences in Condition 4.2(b), we are able to make this argument on both sides to obtain pairs of coefficients $\left(c_{1}^{-}, c_{2}^{-}\right)$and $\left(c_{1}^{+}, c_{2}^{+}\right)$ such that both $c_{1}^{-} \phi+c_{2}^{-} \psi$ and $c_{1}^{+} \phi+c_{2}^{+} \psi$ majorize $g_{r}$ and agree with $g_{r}$ at $x$. Using convex combinations shows that the whole family of coefficients $\left(\lambda c_{1}^{-}+(1-\lambda) c_{1}^{+}, \lambda c_{2}^{-}+(1-\lambda) c_{2}^{+}\right)$, where $0 \leq \lambda \leq 1$, also provide majorizing functions.

(iii) Suppose that $g_{r}$ satisfies Condition 4.2(b) at $x$ with a ' $<$ ' inequality. The same proof as in case (b)(ii) applies to establish that $c_{1}^{-} \phi+c_{2}^{-} \psi$ majorizes $g_{r}$, with equality at $x$. However, $c_{1}^{-} \phi+c_{2}^{-} \psi$ is smooth at $x$ so

$$
\left[c_{1}^{-} \phi+c_{2}^{-} \psi\right]^{\prime}(x) \leq \liminf _{y \nearrow x} \frac{g_{r}(y)-g_{r}(x)}{y-x}<\limsup _{z \searrow x} \frac{g_{r}(z)-g_{r}(x)}{z-x} .
$$

Thus, $\left[c_{1}^{-} \phi+c_{2}^{-} \psi\right](z)<g_{r}(z)$ for some $z>x$ sufficiently close to $x$, a contradiction. Hence, $x \in \varsigma^{\circ}$ implies that Condition 4.2(b) can only be satisfied with a ' $>$ ' inequality.

The proof of case (b)(iii) indicates a condition which implies that $x \in \mathcal{C}$. We formalize this result in the following proposition.

Proposition 4.2. Suppose that $g_{r}$ satisfies Conditions 2.1, 4.1, and 4.2. If $x$ is a point at which

$$
\liminf _{y \nearrow x} \frac{g_{r}(y)-g_{r}(x)}{y-x}<\limsup _{z \searrow x} \frac{g_{r}(z)-g_{r}(x)}{z-x}
$$

then $x \in \mathcal{C}$.

An implication of Theorem 4.2 is that the optimal stopping problem has been reformulated as two different optimization problems. We may solve the nonlinear maximization problem $J\left(\tau_{a, b} ; x\right)$ over the values of $a \leq x$ and $b \geq x$. We may also solve the restricted dual LP (3.3) over coefficients $c_{1}$ and $c_{2}$. Only Conditions 2.1, 4.1, and 4.2 are imposed on $g_{r}$ so little regularity is required.

We emphasize the constructive nature of this approach. For each initial position $x$, the optimizing values $a^{*}$ and $b^{*}$ determine an interval $\left[a^{*}, b^{*}\right]$ which may be degenerate. Consider an $x$ for which $a^{*}<x<b^{*}$ so that the interval $\left[a^{*}, b^{*}\right]$ is not degenerate. Then, for each $x \in\left[a^{*}, b^{*}\right]$, the corresponding optimizing values are also given by $a^{*}$ and $b^{*}$. So, for each $x$ in the interval, the coefficients $c_{1}^{*}$ and $c_{2}^{*}$ given by (4.3) are constant. Moreover, on $\left[a^{*}, b^{*}\right]$, $c_{1}^{*} \phi+c_{2}^{*} \psi$ is the minimal harmonic function which majorizes $g_{r}$. Thus, a single optimization determines the value function over the interval;

$$
V_{1}(x)=c_{1}^{*} \phi(x)+c_{2}^{*} \psi(x), \quad x \in\left[a^{*}, b^{*}\right] .
$$

For the degenerate interval $\left[a^{*}, b^{*}\right]=\{x\}$, the proof of Theorem 4.2 shows how to find coefficients $c_{1}^{*}$ and $c_{2}^{*}$ such that $c_{1}^{*} \phi+c_{2}^{*} \psi$ majorizes $g_{r}$ with $c_{1}^{*} \phi(x)+c_{2}^{*} \psi(x)=g_{r}(x)$. 
Thus, the value function can be constructed by solving the family of nonlinear optimization problems or by solving the family of restricted dual LPs (3.3) or by some combination of these approaches.

\subsection{Smooth pasting}

Suppose now that $g_{r}$ has some additional smoothness. Specifically, suppose that $g_{r}$ is $C^{1}$ in a neighborhood of the optimizing values $a^{*}$ and $b^{*}$. For each $a$ and $b$ with $a \leq x \leq b$ and $a<b$, define functions $c_{1}$ and $c_{2}$ by (4.3). Since we are interested in optimizing with respect to $a$ and $b$, we simplify notation by letting $h(a, b)=J\left(\tau_{a, b} ; x\right)=c_{1}(a, b) \phi(x)+c_{2}(a, b) \psi(x)$. Using the smoothness of $g_{r}$, taking partial derivatives with respect to $a$ and $b$ and simplifying the expressions yields

$$
\begin{aligned}
\frac{\partial h}{\partial a}(a, b)= & {\left[g_{r}^{\prime}(a)-\left(\frac{g_{r}(a) \psi(b)-g_{r}(b) \psi(a)}{\phi(a) \psi(b)-\phi(b) \psi(a)} \phi^{\prime}(a)+\frac{g_{r}(b) \phi(a)-g_{r}(a) \phi(b)}{\phi(a) \psi(b)-\phi(b) \psi(a)} \psi^{\prime}(a)\right)\right] } \\
& \times\left[\frac{\phi(x) \psi(b)-\phi(b) \psi(x)}{\phi(a) \psi(b)-\phi(b) \psi(a)}\right] \\
= & {\left[g_{r}^{\prime}(a)-\left(c_{1}(a, b) \phi+c_{2}(a, b) \psi\right)^{\prime}(a)\right]\left[\frac{\phi(x) \psi(b)-\phi(b) \psi(x)}{\phi(a) \psi(b)-\phi(b) \psi(a)}\right] }
\end{aligned}
$$

and

$$
\begin{aligned}
\frac{\partial h}{\partial b}(a, b)= & {\left[g_{r}^{\prime}(b)-\left(\frac{g_{r}(a) \psi(b)-g_{r}(b) \psi(a)}{\phi(a) \psi(b)-\phi(b) \psi(a)} \phi^{\prime}(b)+\frac{g_{r}(b) \phi(a)-g_{r}(a) \phi(b)}{\phi(a) \psi(b)-\phi(b) \psi(a)} \psi^{\prime}(b)\right)\right] } \\
& \times\left[\frac{\phi(a) \psi(x)-\phi(x) \psi(a)}{\phi(a) \psi(b)-\phi(b) \psi(a)}\right] \\
= & {\left[g_{r}^{\prime}(b)-\left(c_{1}(a, b) \phi+c_{2}(a, b) \psi\right)^{\prime}(b)\right]\left[\frac{\phi(a) \psi(x)-\phi(x) \psi(a)}{\phi(a) \psi(b)-\phi(b) \psi(a)}\right] . }
\end{aligned}
$$

Consider the expression on the right-hand side of (4.9). When $x=b$, the second factor is 0 , indicating that there is no change in $h$ as one moves the stopping location $a$. This is intuitively clear since $x=b$ implies that the process is stopped immediately. For $a \leq x<b$, the second factor is strictly positive and less than or equal to 1 . A similar analysis of the right-hand side expression in (4.11) shows that the second factor is 0 when $x=a$, and is strictly positive and bounded by 1 for $a<x \leq b$.

From these observations, we see that setting $h_{a}=0$ and $h_{b}=0$ requires either $a=x$ or, from (4.10),

$$
g_{r}^{\prime}(a)=\left(c_{1}(a, b) \phi+c_{2}(a, b) \psi\right)^{\prime}(a)
$$

and $b=x$ or, from (4.12),

$$
g_{r}^{\prime}(b)=\left(c_{1}(a, b) \phi+c_{2}(a, b) \psi\right)^{\prime}(b) .
$$

Thus, when $a^{*} \neq x$ and $b^{*} \neq x$, the optimization over $a$ and $b$ imposes equality of the first derivatives of the functions $g_{r}$ and $c_{1}(a, b) \phi+c_{2}(a, b) \psi$ at the optimizers. At the beginning of Section 4, it was shown that

$$
g_{r}(a)=\left(c_{1}(a, b) \phi+c_{2}(a, b) \psi\right)(a) \quad \text { and } \quad g_{r}(b)=\left(c_{1}(a, b) \phi+c_{2}(a, b) \psi\right)(b)
$$

for every choice of $a$ and $b$. This means that at an optimal pair $\left(a^{*}, b^{*}\right)$ of stopping locations, 
either $a^{*}=x$ and the process stops immediately or $a^{*}$ satisfies the smooth pasting condition, and, similarly, either $b^{*}=x$ or the smooth pasting condition is satisfied at $b^{*}$.

In the case of natural or entrance-not-exit boundary points, we point out that $a^{*}$ could be $x_{l}$, in which case $J_{r}\left(\tau_{x_{l}, b} ; x\right)$ has expression (2.11) and the smooth pasting condition is only required at $b^{*}$, and, similarly, should $b^{*}=x_{r}$, the expression for $J_{r}\left(\tau_{a, x_{r}} ; x\right)$ is (2.10) and smooth pasting is only required at $a^{*}$.

The above argument assumes that the partial derivatives $\partial h / \partial a$ and $\partial h / \partial b$ actually equal 0 for some $a$ and $b$ with $x_{l} \leq a \leq x$ and $x \leq b \leq x_{r}$. The optimal value could also occur with $a=x_{l}$ or $b=x_{r}$ without either the smooth pasting condition holding or $a^{*}$ or $b^{*}$ being $x$. The endpoints must also be considered when determining the optimizing values of $a$ and $b$.

\section{Unrestricted stopping problem}

This section extends the optimality of the two-point stopping rule to the unrestricted problem which optimizes $J_{r}(\tau ; x)$ over $\tau \in \mathcal{A}$. The extension requires an additional assumption on $g_{r}$. Observe that $g_{r}=f_{r}+g$ in which $f_{r}$ satisfies Condition 1.1. Whereas it may be reasonable to assume that the payoff function $g$ at the stopping location is nonnegative, the solution $f_{r}$ depends on the running reward function $r$, so imposing nonnegativity on $f_{r}$ will be too restrictive. Let $g_{r}^{+}$and $g_{r}^{-}$denote the positive and negative parts of $g_{r}$, respectively.

Condition 5.1. The family of random variables $\left\{\mathrm{e}^{-\alpha \tau} g_{r}^{-}(X(\tau)) \mathbf{1}_{\{\tau<\infty\}}: \tau \in \mathcal{A}_{1}\right\}$ is uniformly integrable.

Remark 5.1. Condition 5.1 involves the process $X$ and the stopping times $\tau$ as well as knowledge of $g_{r}^{-}$, so may be difficult to check. However, a sufficient condition on the function $g_{r}$ under which Condition 5.1 is readily verifiable is that $g_{r}$ be bounded below.

The following theorem demonstrates that the values of the unrestricted and restricted stopping problems are the same.

Theorem 5.1. Assume that Conditions 2.1, 4.1, and 5.1 hold. Then

$$
V_{1}(x)=\sup _{\tau \in \mathscr{A}_{1}} J_{r}(\tau ; x)=\sup _{\tau \in \mathcal{A}} J_{r}(\tau ; x)=V_{r}(x) .
$$

Proof. We prove that the result in the event $x_{l}$ is either a natural or entrance-not-exit boundary point and that $x_{r}$ is an exit-not-entrance boundary point; the argument is symmetric when the classifications of $x_{l}$ and $x_{r}$ are reversed and easily combine when both are natural or entrancenot-exit.

Select $\tau \in \mathcal{A}$ arbitrarily. Let $\left\{a_{n}\right\}$ be a strictly decreasing sequence with $a_{n} \searrow x_{l}$. Let $\tau_{a_{n}}=$ $\inf \left\{t \geq 0: X(t)=a_{n}\right\}$. Observe that, for each $n, \tau_{a_{n}} \in \mathcal{A}_{1}$ and, furthermore, $\tau \wedge \tau_{a_{n}} \in \mathcal{A}_{1}$. It therefore follows that, for each $n$,

$$
\begin{aligned}
V_{1}(x) \geq & \mathrm{E}\left[\mathrm{e}^{-\alpha\left(\tau \wedge \tau_{a_{n}}\right)} g_{r}\left(X\left(\tau \wedge \tau_{a_{n}}\right)\right) \mathbf{1}_{\left\{\tau \wedge \tau_{\left.a_{n}<\infty\right\}}\right]}\right] \\
= & \mathrm{E}\left[\mathrm{e}^{-\alpha\left(\tau \wedge \tau_{a_{n}}\right)} g_{r}^{+}\left(X\left(\tau \wedge \tau_{a_{n}}\right)\right) \mathbf{1}_{\left\{\tau \wedge \tau_{a_{n}}<\infty\right\}}\right]-\mathrm{E}\left[\mathrm{e}^{-\alpha\left(\tau \wedge \tau_{a_{n}}\right)} g_{r}^{-}\left(X\left(\tau \wedge \tau_{a_{n}}\right)\right) \mathbf{1}_{\left\{\tau \wedge \tau_{a_{n}}<\infty\right\}}\right] \\
\geq & \mathrm{E}\left[\mathrm{e}^{-\alpha\left(\tau \wedge \tau_{a_{n}}\right)} g_{r}^{+}\left(X\left(\tau \wedge \tau_{a_{n}}\right)\right) \mathbf{1}_{\left\{\tau \wedge \tau_{a_{n}}<\infty\right\}} \mathbf{1}_{\{\tau<\infty\}}\right] \\
& -\mathrm{E}\left[\mathrm{e}^{-\alpha\left(\tau \wedge \tau_{a_{n}}\right)} g_{r}^{-}\left(X\left(\tau \wedge \tau_{a_{n}}\right)\right) \mathbf{1}_{\left\{\tau \wedge \tau_{\left.a_{n}<\infty\right\}}<\mathbf{1}_{\{\tau<\infty\}}\right]}\right. \\
& -\mathrm{E}\left[\mathrm{e}^{-\alpha\left(\tau \wedge \tau_{a_{n}}\right)} g_{r}^{-}\left(X\left(\tau \wedge \tau_{a_{n}}\right)\right) \mathbf{1}_{\left\{\tau \wedge \tau_{a_{n}}<\infty\right\}} \mathbf{1}_{\{\tau=\infty\}}\right]
\end{aligned}
$$


The second inequality is justified since the integrand in the first expectation is nonnegative and is being integrated over a smaller set. Observe that on the set $\{\tau<\infty\}, \tau \wedge \tau_{a_{n}} \rightarrow \tau$ since $\tau_{a_{n}} \rightarrow \infty$ a.s.; in fact, for each $\omega \in\{\tau<\infty\}, \tau(\omega) \wedge \tau_{a_{n}}(\omega)=\tau(\omega)$ for sufficiently large $n$, and, hence, again, for sufficiently large $n$,

$$
\begin{aligned}
& \mathrm{e}^{-\alpha\left(\tau(\omega) \wedge \tau_{a_{n}}(\omega)\right)} g_{r}^{+}\left(X\left(\tau(\omega) \wedge \tau_{a_{n}}(\omega), \omega\right)\right) \mathbf{1}_{\left\{\tau \wedge \tau_{a_{n}}<\infty\right\}} \mathbf{1}_{\{\tau<\infty\}} \\
& =\mathrm{e}^{-\alpha \tau(\omega)} g_{r}^{+}(X(\tau(\omega), \omega)) \mathbf{1}_{\{\tau<\infty\}}
\end{aligned}
$$

Thus,

$$
\lim _{n \rightarrow \infty} \mathrm{e}^{-\alpha\left(\tau \wedge \tau_{a_{n}}\right)} g_{r}^{+}\left(X\left(\tau \wedge \tau_{a_{n}}\right)\right) \mathbf{1}_{\left\{\tau \wedge \tau_{a_{n}}<\infty\right\}} \mathbf{1}_{\{\tau<\infty\}}=\mathrm{e}^{-\alpha \tau} g_{r}^{+}(X(\tau)) \mathbf{1}_{\{\tau<\infty\}} \quad \text { a.s. },
$$

with the same result holding for $g_{r}^{-}$replacing $g_{r}^{+}$. Now consider the last expectation in (5.1). On the event $\{\tau=\infty\}, \tau \wedge \tau_{a_{n}}=\tau_{a_{n}}$, so this expectation becomes

$$
\mathrm{E}\left[\mathrm{e}^{-\alpha \tau_{a_{n}}} g_{r}^{-}\left(X\left(\tau_{a_{n}}\right)\right) \mathbf{1}_{\left\{\tau_{a_{n}}<\infty\right\}} \mathbf{1}_{\{\tau=\infty\}}\right]
$$

We claim that (5.2) converges to 0 as $n \rightarrow \infty$. To see this, observe that the integrand is nonnegative, so we have

$$
\begin{aligned}
\mathrm{E}\left[\mathrm{e}^{-\alpha \tau_{a_{n}}} g_{r}^{-}\left(X\left(\tau_{a_{n}}\right)\right) \mathbf{1}_{\left\{\tau_{a_{n}}<\infty\right\}} \mathbf{1}_{\{\tau=\infty\}}\right] & \leq \mathrm{E}\left[\mathrm{e}^{-\alpha \tau_{a_{n}}} g_{r}^{-}\left(X\left(\tau_{a_{n}}\right)\right)\right] \\
& =g_{r}^{-}\left(a_{n}\right) \mathrm{E}\left[\mathrm{e}^{-\alpha \tau_{a_{n}}}\right] \\
& =g_{r}^{-}\left(a_{n}\right) \frac{\phi(x)}{\phi\left(a_{n}\right)} .
\end{aligned}
$$

By Condition 4.1(b), this upper bound converges to 0 as $n \rightarrow \infty$. Returning to the examination of (5.1), Condition 5.1 implies that $\left\{\mathrm{e}^{-\alpha\left(\tau \wedge \tau_{a_{n}}\right)} g_{r}^{-}\left(X\left(\tau \wedge \tau_{a_{n}}\right)\right) \mathbf{1}_{\left\{\tau \wedge \tau_{a_{n}}<\infty\right\}} \mathbf{1}_{\{\tau<\infty\}}\right\}$ is uniformly integrable. Hence, applying Fatou's Lemma yields

$$
\begin{aligned}
V_{1}(x) \geq & \liminf _{n \rightarrow \infty} \mathrm{E}\left[\mathrm{e}^{-\alpha\left(\tau \wedge \tau_{a_{n}}\right)} g_{r}^{+}\left(X\left(\tau \wedge \tau_{a_{n}}\right)\right) \mathbf{1}_{\left\{\tau \wedge \tau_{a_{n}}<\infty\right\}}\right] \\
& -\lim _{n \rightarrow \infty} \mathrm{E}\left[\mathrm{e}^{-\alpha\left(\tau \wedge \tau_{a_{n}}\right)} g_{r}^{-}\left(X\left(\tau \wedge \tau_{a_{n}}\right)\right) \mathbf{1}_{\left\{\tau \wedge \tau_{a_{n}}<\infty\right\}}\right] \\
\geq & \mathrm{E}\left[\mathrm{e}^{-\alpha \tau} g_{r}^{+}(X(\tau)) \mathbf{1}_{\{\tau<\infty\}}\right]-\mathrm{E}\left[\mathrm{e}^{-\alpha \tau} g_{r}^{-}(X(\tau)) \mathbf{1}_{\{\tau<\infty\}}\right] \\
= & \mathrm{E}\left[\mathrm{e}^{-\alpha \tau} g_{r}(X(\tau)) \mathbf{1}_{\{\tau<\infty\}}\right] .
\end{aligned}
$$

Taking the supremum over $\tau \in \mathcal{A}$ demonstrates $V_{1}(x) \geq V_{r}(x)$. Since $\mathcal{A}_{1} \subset \mathcal{A}$, the opposite inequality follows immediately, establishing the result.

\section{Examples}

In this section we illustrate how to construct the value function $V$ using the nonlinear optimization method, the restricted dual LP, and a combination of these approaches. We consider a geometric Brownian motion process for these examples. This choice of diffusion implies that $x_{l}=0$ and $x_{r}=\infty$, and that both boundaries are natural. Additional examples involving other types of processes may be found in [9], though for those examples the initial position is assumed to be small. For later reference, we begin by determining the important results concerning geometric Brownian motion. 
Let $\alpha>0$ denote the discount rate, and let $\mu \leq \alpha$ and $\sigma>0$ be constants. We assume that the process $X$ satisfies the stochastic differential equation

$$
\mathrm{d} X(t)=\mu X(t) \mathrm{d} t+\sigma X(t) \mathrm{d} W(t), \quad X(0)=x>0 .
$$

The generator $A$ of the process $X$ is $A f(y)=\left(\sigma^{2} / 2\right) y^{2} f^{\prime \prime}(y)+\mu y f^{\prime}(y)$, so the solutions of the differential equation (1.4) are $\phi(y)=y^{\gamma_{1}}$ and $\psi(y)=y^{\gamma_{2}}$, in which

$$
\gamma_{1}:=\frac{1}{2}-\frac{\mu}{\sigma^{2}}-\sqrt{\left(\frac{1}{2}-\frac{\mu}{\sigma^{2}}\right)^{2}+\frac{2 \alpha}{\sigma^{2}}}<0<\frac{1}{2}-\frac{\mu}{\sigma^{2}}+\sqrt{\left(\frac{1}{2}-\frac{\mu}{\sigma^{2}}\right)^{2}+\frac{2 \alpha}{\sigma^{2}}}=: \gamma_{2} .
$$

Consider a general solution $f=c_{1} \phi+c_{2} \psi$ in which $c_{1}, c_{2}>0$. Evaluating the derivative we have $f^{\prime}(y)=\gamma_{1} c_{1} y^{\left(\gamma_{1}-1\right)}+\gamma_{2} c_{2} y^{\left(\gamma_{2}-1\right)}$. Setting $f^{\prime}=0$ and solving for $y$ yields

$$
y_{c}=\left(\frac{-c_{1} \gamma_{1}}{c_{2} \gamma_{2}}\right)^{1 /\left(\gamma_{2}-\gamma_{1}\right)} .
$$

Observe that $f^{\prime \prime}(y)>0$ for $y>0$ since $\alpha \geq \mu$ implies that $\gamma_{2} \geq 1$. Thus, $f^{\prime}$ is strictly decreasing for $y<y_{c}$ and strictly increasing for $y>y_{c}$, and, hence, $y_{c}$ is a minimizer of $f$.

We utilize this structure of $f$.

Example 6.1. (Perpetual put option.) For this example, $X$ represents the price of a risky asset in a Black-Scholes market. Let $K>0$ denote the option's strike price. The goal is to select a stopping time $\tau$ so as to maximize

$$
\mathrm{E}\left[\mathrm{e}^{-\alpha \tau}(K-X(\tau))^{+}\right]
$$

For this optimization problem to give the risk-neutral price of the option, $\alpha$ is the interest rate on the nonrisky asset and the expectation is taken with respect to the risk-neutral measure with the result that the mean rate of return of the risky asset is $\mu=\alpha$. As a result, $\gamma_{1}=-2 \alpha / \sigma^{2}$ and $\gamma_{2}=1$.

This optimal stopping problem has no running reward $r$; a reward $g$ is only earned when the option is exercised at the stopping time. The reward function is $g(y)=(K-y)^{+}$. Our goal is, for each $x \in(0, \infty)$, to maximize (2.9) over stopping locations $a$ and $b$ with $a \leq x \leq b$.

We note that, since $r=0, J_{r}=J$, so we drop the subscript for both $J$ and $\widehat{J}$ in the discussion of this example.

Solution 1: maximizing $J\left(\tau_{a, b} ; x\right)$. Initial analysis of stopping location a to the left of $x$. Consider the case when $x>K$. For any stopping location $a$ with $a>K$ and, hence, any $b$ with $b>K$ as well, $J\left(\tau_{a, b} ; x\right)=0$ by simple evaluation of (2.9) since $g(a)=0=g(b)$. But, for each $a \in(0, K), J\left(\tau_{a, b} ; x\right)>0$, so the optimal choice $a^{*}$ must be less than $K$.

Analysis of stopping location $b$ to the right of $x$. Now consider the case $x>0$, and let $a<$ $x \wedge K$. First consider the case when $x<b<K$. The function $J_{a, b}$ having expression (4.2) but which takes the independent variable in $\left[x_{l}, x_{r}\right]$ is strictly convex and satisfies $J_{a, b}(a)=K-a$ and $J_{a, b}(b)=K-b$, and, hence, it follows that $J_{a, b}(x)<K-x=g_{r}(x)$.

Now consider any $b>K \vee x$ and the stopping time $\tau_{a, b}$. The second summand in (2.9) is 0 and the first summand depends only on $b$ in its mass. In fact, this mass is an increasing function of $b$ and, hence, the maximal value occurs when $b=\infty$. This means that an optimal stopping rule has the form $\tau_{a}$ which exercises the option when the stock price first hits some value $a$. The goal now reduces to maximizing $J\left(\tau_{a} ; x\right)$ given by (2.10) over values of $a \in[0, K]$. 


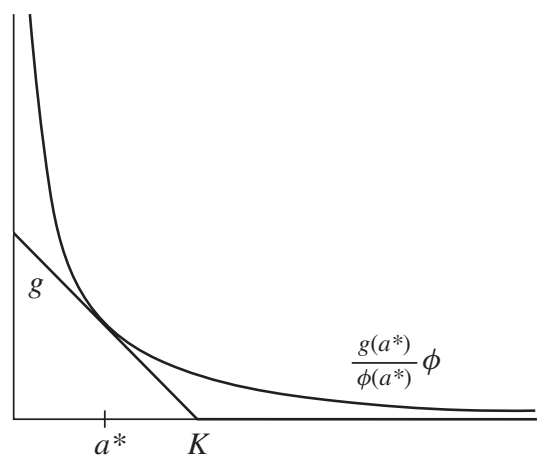

(a)

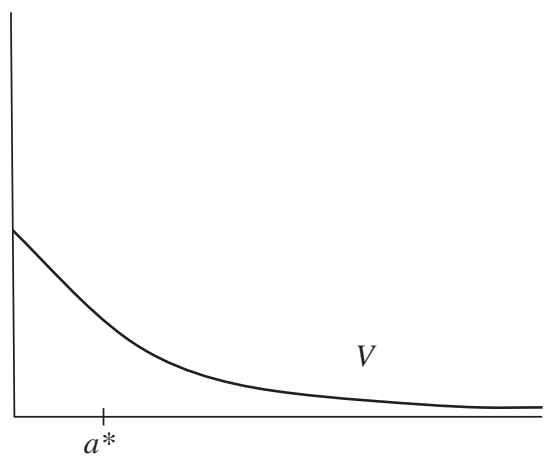

(b)

FiguRE 1: Relation between $g$ and $\left(g\left(a^{*}\right) / \phi\left(a^{*}\right)\right) \phi$ and the value function $V$.

Fix $x>0$, and let $h(a)=(g(a) / \phi(a)) \phi(x)=\left(K a^{-\gamma_{1}}-a^{1-\gamma_{1}}\right) x^{\gamma_{1}}$ for $0<a<K$. Setting $h^{\prime}(a)=0$ results in $a^{*}=-K \gamma_{1} /\left(1-\gamma_{1}\right)$. A simple analysis shows that $a^{*}$ is a maximizer. This means that, for any $x \geq a^{*}$, an optimal stopping rule is given by $\tau_{a^{*}}$. The relationship between the functions $g$ and $\left(g\left(a^{*}\right) / \phi\left(a^{*}\right)\right) \phi$ is displayed in Figure 1(a).

Analysis when $x<a^{*}$. Now consider $0<x<a^{*}$. Suppose that there is an optimal stopping location $a_{1}<x<a^{*}$. Since, for $0<y<K, g$ is continuously differentiable, $a_{1}$ would satisfy the smooth pasting principle. However, $a^{*}$ is the unique value at which $(g(a) / \phi(a)) \phi^{\prime}(a)=-1=g^{\prime}(a)$. Thus, for no $a_{1}<x<a^{*}$ is $a_{1}$ an optimal stopping location. It then follows that the optimal stopping rule is to stop immediately.

The value function $V$ and optimal stopping time $\tau^{*}$ are given by

$$
V(x)=\left\{\begin{array}{ll}
K-x & \text { for } 0 \leq x \leq a^{*}, \\
\left(K-a^{*}\right)\left(\frac{x}{a^{*}}\right)^{\gamma_{1}} & \text { for } x \geq a^{*},
\end{array} \quad \text { and } \quad \tau^{*}= \begin{cases}\tau_{x}=0 & \text { for } x \leq a^{*} \\
\tau_{a^{*}} & \text { for } x \geq a^{*}\end{cases}\right.
$$

The value function $V$ is displayed in Figure 1(b).

Solution 2: minimizing $\widehat{J}\left(c_{1}, c_{2} ; g_{r}\right)$. We demonstrate how to use the restricted dual LP (3.3) to obtain the value function. Let $x>0$ be fixed. First observe that the majorizing condition of $c_{1} \phi+c_{2} \psi$ over $g$ along with $\phi(0+)=\infty$ and the strict positivity of $g$ near 0 implies that $c_{1}$ must be positive. Also, since $\psi(\infty)=\infty$ and $\phi(\infty)=0, c_{2}$ must also be nonnegative. Since $\psi(x)>0$, the objective function would be minimized if $c_{2}=0$. In this case, the majorizing condition reduces to $c_{1} \phi \geq g$, yielding $c_{1}^{*}=\sup _{0<a \leq x} g(a) / \phi(a)$. Observe that $a^{*}=\left(-\gamma_{1} /\left(1-\gamma_{1}\right)\right) K$ is the optimizer. Thus, for any initial value $x \geq a^{*}$, the pair $\left(c_{1}^{*}, 0\right)$ provides the optimal solution for the restricted dual LP.

For $x<a^{*}, c_{2}$ must be positive. In trying to minimize $\left[c_{1} \phi+c_{2} \psi\right](x)$ subject to the majorizing condition, we must have $\left[c_{1} \phi+c_{2} \psi\right](x) \geq g(x)$. The question then arises as to whether it is possible to have $\left[c_{1} \phi+c_{2} \psi\right](x)=g(x)$. Since $g$ is $C^{1}$ on $\left(0, a^{*}\right)$, the majorizing requirement implies that $\left[c_{1} \phi+c_{2} \psi\right]^{\prime}(x)=g^{\prime}(x)$ when $\left[c_{1} \phi+c_{2} \psi\right](x)=g(x)$. This then sets up the system (4.4) of linear equations that determine the optimal choice of $c_{1}^{*}$ and $c_{2}^{*}$ given by (4.5). Since $\left[c_{1}^{*} \phi+c_{2}^{*} \psi\right]^{\prime \prime}>0,\left[c_{1}^{*} \phi+c_{2}^{*} \psi\right]^{\prime}$ is strictly increasing and it follows that $c_{1}^{*} \phi+c_{2}^{*} \psi$ majorizes $g$. 
Example 6.2. (Forest harvest with carbon credits.) Let $X$ satisfy (6.1) with $\mu, \sigma>0$. The process $X$ now represents the quantity of lumber in a stand of forest. When the stand is harvested, it earns a net profit of $g(y)=k_{1} y^{\beta}-k_{2}$, in which $k_{1}, k_{2}>0$ and $\beta>0$. Until harvest, the owner is paid a carbon credit that is proportional to the same power of the size of the forest, so $r(y)=R y^{\beta}$. The owner's objective is to select a stopping time $\tau$ so as to maximize

$$
\mathrm{E}\left[\int_{0}^{\tau} \mathrm{e}^{-\alpha t} R X^{\beta}(t) \mathrm{d} t+\mathrm{e}^{-\alpha \tau}\left(k_{1} X^{\beta}(\tau)-k_{2}\right)\right] .
$$

We make the following assumptions about the relation between the parameters. In order to have a finite maximum in (6.3), we impose the condition that $\beta<\gamma_{2}$, where $\gamma_{2}$ is defined in (6.2); otherwise, the owner can receive arbitrarily large discounted rewards by choosing to stop when $X$ hits sufficiently large values. The assumption that $0<\beta<\gamma_{2}$ also implies that $\left(\sigma^{2} / 2\right) \beta(\beta-1)+\mu \beta-\alpha<0$. In addition, we assume that $k_{1}\left[\alpha-\beta \mu-\left(\sigma^{2} / 2\right) \beta(\beta-1)\right]>R$, which will imply the existence of a finite optimal stopping time.

Applying the differential operator $A f-\alpha f$ to the function

$$
f_{r}(y)=\frac{R}{\left(\sigma^{2} / 2\right) \beta(\beta-1)+\mu \beta-\alpha} y^{\beta}
$$

results in $A f_{r}-\alpha f_{r}=R y^{\beta}$. Let $k_{3}=k_{1}+R /\left(\left(\sigma^{2} / 2\right) \beta(\beta-1)+\mu \beta-\alpha\right)$. Thus, the function $g_{r}(y)=f_{r}(y)+g(y)=k_{3} y^{\beta}-k_{2}$. We note that the assumptions on the parameters imply that $k_{3}>0$, so that the owner has an incentive to harvest the lumber at some point. It then follows that $g_{r}$ is strictly increasing, $g_{r}(0)=-k_{2}$ and $g_{r}\left(y_{0}\right)=0$ for $y_{0}=\left(k_{2} / k_{3}\right)^{1 / \beta}$. For $x<y_{0}$, choosing any value $a$ such that $0<a<x$ has value $J\left(\tau_{a, b} ; a\right)=g_{r}(a)<0$. Selecting $a=0$ and $b \geq y_{0}$ and, hence, $c_{1}(a, b)=0$, however, yields

$$
J\left(\tau_{a, b} ; x\right)=J\left(\tau_{b} ; x\right)=\frac{g_{r}(b)}{\psi(b)} x^{\gamma_{2}},
$$

with the result that its value is nonnegative for all $x \geq 0$. Thus, for sufficiently small $x$, the optimal stopping time will be $\tau_{b}$ for some $b>x \vee y_{0}$. We now seek the optimal value of $b$.

Define $h(b)=g_{r}(b) / \psi(b)=k_{3} b^{\beta-\gamma_{2}}-k_{2} b^{-\gamma_{2}}$. Setting the derivative of $h$ equal to 0 yields

$$
0=k_{3}\left(\beta-\gamma_{2}\right) b^{\beta-\gamma_{2}-1}+k_{2} \gamma_{2} b^{-\gamma_{2}-1}=\left[k_{3}\left(\beta-\gamma_{2}\right) b^{\beta}+k_{2} \gamma_{2}\right] b^{-\gamma_{2}-1},
$$

and, hence, a unique maximum occurs at $b^{*}=\left(k_{2} \gamma_{2} / k_{3}\left(\gamma_{2}-\beta\right)\right)^{1 / \beta}$.

Now consider the situation for $x \in\left(b^{*}, \infty\right)$. Since, for $x>b^{*}, g_{r} \in C^{1}\left(b^{*}, \infty\right)$. If there were two distinct points $a^{*}$ and $b^{*}$ for which the stopping time $\tau_{a^{*}, b^{*}}$ would be optimal, the points would need to satisfy the smooth pasting conditions (4.13) and (4.14). These conditions would imply that $g_{r}^{\prime}(y)=c_{1} \phi^{\prime}(y)+c_{2} \psi^{\prime}(y)$ for at least two values of $y$. Differentiating

$$
h_{1}(y)=\frac{c_{1} \phi^{\prime}(y)+c_{2} \psi^{\prime}(y)}{g_{r}^{\prime}(y)}=\frac{c_{1} \gamma_{1}}{k_{3} \beta} y^{\gamma_{1}-\beta}+\frac{c_{2} \gamma_{2}}{k_{3} \beta} y^{\gamma_{2}-\beta}
$$

yields

$$
h_{1}^{\prime}(y)=\frac{c_{1} \gamma_{1}\left(\gamma_{1}-\beta\right)}{k_{3} \beta} y^{\gamma_{1}-\beta-1}+\frac{c_{2} \gamma_{2}\left(\gamma_{2}-\beta\right)}{k_{3} \beta} y^{\gamma_{2}-\beta-1}>0 .
$$


The ratio $\left(c_{1} \phi^{\prime}+c_{2} \psi^{\prime}\right) / g_{r}^{\prime}$ is therefore strictly increasing and $b^{*}$ is the only value which satisfies the smooth pasting principle. Thus, for $x>b^{*}$, there cannot be two distinct optimal points $a^{*}$ and $b^{*}$. The only optimal stopping rule is $\tau^{*}=0$.

The value function is therefore

$$
V_{r}(x)= \begin{cases}\left(\frac{g_{r}\left(b^{*}\right)}{\left(b^{*}\right)_{2}}\right) x^{\gamma_{2}} & \text { for } x \leq b^{*} \\ k_{3} x-k_{2} & \text { for } x \geq b^{*}\end{cases}
$$

The value function for the original stopping problem is

$$
V(x)= \begin{cases}\left(\frac{g_{r}\left(b^{*}\right)}{\left(b^{*}\right)^{\gamma_{2}}}-\frac{R}{\left(\sigma^{2} / 2\right) \beta(\beta-1)+\mu \beta-\alpha}\right) x^{\gamma_{2}} & \text { for } x \leq b^{*}, \\ k_{1} x^{\beta}-k_{2} & \text { for } x \geq b^{*} .\end{cases}
$$

\section{Concluding remarks}

In this paper we established two complementary optimization approaches to the solution of optimal stopping problems for one-dimensional diffusions. One method recast the problem as a nonlinear maximization over two-point stopping locations while the other determined a semi-infinite linear program over the coefficients of the harmonic functions. The combination of an explicit formula for the expected reward obtained using a two-point hitting rule and duality analysis proved that the class of such two-point hitting times contains an optimal stopping rule.

The method is local in nature in that the optimization problems are parameterized by the initial position $x$ of the diffusion. Therefore, in principle, it is necessary to solve the entire family of optimization problems in order to determine the value function. In practice, however, the structure of the two-point stopping rules determines the value function over intervals of initial values. Strong duality between the optimization problems allows us to choose whichever problem is easier to analyze for a given initial value.

The restricted dual linear program (3.3) is quite similar to the approach of Shiryaev [15] in that it seeks a minimal harmonic function which majorizes the terminal reward function. Shiryaev's approach seeks a minimal super-harmonic function for all values of $x$ since this function is the value function $V$. Our approach only determines the value function piecewise, so is able to utilize the fundamental solutions of the differential equation $A f-\alpha f=0$ to characterize all harmonic functions. When the pieces are connected, the resulting value function is, of course, superharmonic.

\section{Acknowledgement}

The authors would like to express appreciation to the anonymous referee for careful reading of the manuscript, constructive suggestions, and a clear argument that shows how to rewrite running rewards as terminal rewards. The manuscript has greatly improved as a result.

\section{References}

[1] Alili, L. And Kyprianou, A. E. (2005). Some remarks on first passage of Lévy processes, the American put and smooth pasting principles. Ann. Appl. Prob. 15, 2062-2080.

[2] Alvarez, L. H. R. (2001). Reward functionals, salvage values, and optimal stopping. Math. Meth. Operat. Res. 54, 315-337.

[3] Borodin, A. N. and Salminen, P. (2002). Handbook of Brownian Motion-Facts and Formulae, 2nd edn. Birkhäuser, Basel. 
[4] Dayanik, S. and Karatzas, I. (2003). On the optimal stopping problem for one-dimensional diffusions. Stoch. Process. Appl. 107, 173-212.

[5] Dynkin, E. B. (1965). Markov Processes, Vol. 2. Springer, New York.

[6] El Karoui, N. (1981). Les aspects probabilistes du contrôle stochastique. In Lecture Notes in Math. 876, Springer, Berlin, pp. 73-238.

[7] Ethier, S. N. And Kurtz, T. G. (1986). Markov Processes. John Wiley, New York.

[8] Helmes, K. AND StockBridge, R. H. (2007). Linear programming approach to the optimal stopping of singular stochastic processes. Stochastics 79, 309-335.

[9] Helmes, K. And Stockbridge, R. H. (2010). Thinning and harvesting in stochastic forest models. Preprint.

[10] Itô, K. And McKean, H. P., JR. (1974). Diffusion Process and Their Sample Paths. Springer, New York.

[11] ØKSEndal, B. (2003). Stochastic Differential Equations, 6th edn. Springer, Berlin.

[12] Peskir, G. And Shiryaev, A. N. (2006). Optimal Stopping and Free-Boundary Problems. Birkhäuser, Basel.

[13] Rogers, L. C. G. AND Williams, D. (2000). Diffusions, Markov Processes and Martingales, Vol. 2. Cambridge University Press.

[14] Salminen, P. (1985). Optimal stopping of one-dimensional diffusions. Math. Nachr. 124, 85-101.

[15] Shiryaev, A. N. (1978). Optimal Stopping Rules. Springer, New York.

[16] ZABCZYK, J. (1979). Introduction to the theory of optimal stopping. In Stochastic Control Theory and Stochastic Differential Systems (Bad Honnef, 1979; Lecture Notes Control Inf. Sci. 16), Springer, Berlin, pp. 227-250. 